\title{
DU VOTE PROFESSIONNEL À LA GRÈVE
}

\section{LES INÉGALITÉS DE PARTICIPATION EN ENTREPRISE ${ }^{1}$}

\section{Pierre Blavier, Tristan Haute, Étienne Pénissat}

En France, ces quinze dernières années, la sociologie du syndicalisme, des relations professionnelles et de l'action collective a développé de nombreuses enquêtes et analyses sur les modes d'action et de mobilisation des salariés dans les entreprises privées et dans la fonction publique $^{2}$. Plusieurs de ces travaux, appuyés par de nouvelles enquêtes statistiques du ministère du Travail, se sont notamment focalisés sur des modes d'action revendicatifs, tels que la grève, en insistant sur les contextes économiques, organisationnels et politiques qui favorisent ou non le recours à de telles pratiques ${ }^{3}$. Ils ont souligné plusieurs changements importants dans les formes de mobilisation en entreprise : un déclin des grèves massives et interprofessionnelles, le maintien à des niveaux élevés de grèves d'entreprises localisées, en particulier dans un contexte de crise économique avec de nombreux plans de licenciements, et la diversification des formes de mobilisation revendicative en dehors des arrêts de travail (manifestations, pétitions, refus collectifs d'heures supplémentaires... $)^{4}$. Des travaux ont également souligné le rôle des stratégies patronales de contre-mobilisation, en particulier les différentes formes de discrimination et de répression de l'action syndicale ${ }^{5}$, qui rendent plus coûteuses la participation et la mobilisation collective des salariés en entreprise. Cette fragilité de la participation en

\footnotetext{
${ }^{1}$ Ce travail s'est nourri de discussions aux XIV et $X^{\mathrm{e}}$ e Congrès de l'Association française de science politique (AFSP) (Montpellier, juillet 2017 ; Bordeaux, juillet 2019), aux Journées internationales de sociologie du travail (Paris, juillet 2018) et au sein des projets de recherche DIALSOC et REGTRAV (Dares) ainsi que CitIndus (ANR-18-CE-26-0019).

${ }^{2}$ Pour une synthèse récente, voir Baptiste Giraud, Karel Yon, Sophie Béroud, Sociologie politique du syndicalisme. Introduction à l'analyse sociologique des syndicats, Paris, Armand Colin, 2018.

${ }^{3}$ Sophie Béroud et al., La lutte continue ? Les conflits du travail dans la France contemporaine, Bellecombe-enBauges, Éditions du Croquant, 2008.

${ }^{4}$ Ibid.

${ }^{5}$ Étienne Pénissat (dir.), « Réprimer et domestiquer : stratégies patronales », Agone, 50, 2013 ; Thomas Amossé, Jean-Michel Denis, « La discrimination syndicale : une discrimination comme les autres ? Enjeux et état des lieux », Travail et Emploi, 145, 2016, p. 5-30.
} 
entreprise $^{6}$ se retrouve en matière électorale, alors même que plusieurs lois relatives au «dialogue social» ont fait des élections professionnelles un enjeu déterminant dans la définition de la représentativité des syndicats et dans la négociation d'entreprise ${ }^{7}$. Ces derniers constats ont d'ailleurs conduit à poser la question des formes d'articulation entre participation dans et hors l'entreprise : les mobilisations récentes autour des occupations de place, comme en Espagne ou en France avec « Nuit Debout ${ }^{8} »$ ou des blocages de ronds-points avec les Gilets jaunes ${ }^{9}$ sont-elles l'expression d'une dévitalisation des modes d'action privilégiés par les syndicats pour protester dans l'entreprise ? Cette question des formes d'articulation n'est pas restreinte aux grèves et aux manifestations. Dans une autre perspective, une importante littérature au niveau international s'est ainsi attachée à mettre à l'épreuve l'existence d'articulations entre la participation au travail et la participation politique dans le champ électoral. Des travaux récents ont par exemple souligné que l'adhésion syndicale favorise la participation électorale lors des scrutins politiques, que ce soit aux États-Unis ${ }^{10}$ ou en Europe ${ }^{11}$.

Si ces travaux permettent, dans le cas français, d'analyser les transformations de la morphologie des modes d'action en entreprise, ils renseignent rarement, à l'exception de ceux sur la syndicalisation ${ }^{12}$ et sur l'occupation de fonctions de représentants du personnel ${ }^{13}$, sur les inégalités de participation en entreprise. Autrement dit, ces pratiques sont assez peu étudiées au prisme des caractéristiques sociales des salariés alors même que nous savons, à partir

${ }^{6}$ Sur cette notion, voir Sophie Béroud, «Perspectives critiques sur la participation dans le monde du travail : éléments de repérage et de discussion », Participations, 5, 2013, p. 5-32.

7 Tristan Haute, « Les salarié.e.s aux urnes. Contribution à l'étude des ressorts collectifs et individuels des votes des salariés aux scrutins professionnels dans le secteur privé en France », thèse de doctorat en science politique, Jean-Gabriel Contamin, Karel Yon (dir.), université de Lille, 2019.

${ }^{8}$ Collectif d'enquête, « Déclassement sectoriel et rassemblement public : éléments de sociographie de Nuit Debout place de la République », Revue française de science politique, 67 (4), août 2017, p. 675-693.

${ }^{9}$ Sur ce débat, voir, par exemple, Laurent Jeanpierre, In Girum. Les leçons politiques des ronds-points, Paris, La Découverte, 2019 ; et Karel Yon, « Le syndicalisme, la retraite et les grèves », Contretemps, 4 mai 2020 , en ligne : www.contretemps.eu/syndicalisme-retraites-greves/.

${ }^{10}$ Jasmine Kerrissey, Evan Schofer, «Union Membership and Political Participation in The United States », Social Forces, 91 (3), 2013, p. 895-928.

${ }^{11}$ Alex Bryson et al., «What Accounts for The Union Member Advantage in Voter Turnout ? Evidence from The European Union, 2002-2008 », Relations industrielles/Industrial Relations, 69 (4), 2014, p. 732-765.

${ }^{12}$ Maria-Teresa Pignoni, « La syndicalisation en France », Dares Analyses, 25, mai 2016.

${ }^{13}$ Thomas Breda, Les représentants du personnel, Paris, Presses de Sciences Po, 2016. 
d'enquêtes dans d'autres espaces de participation comme le champ politique ${ }^{14}$ ou associatif ${ }^{15}$, qu'elles jouent fortement sur les conditions de possibilités à participer sur son lieu de travail. Ceci s'explique en partie par le fait que, jusqu'à récemment, les enquêtes de la statistique publique ne renseignaient pas sur les déterminants individuels de la participation en entreprise, les données publiques étant d'abord focalisées sur la récurrence des modes d'action eux-mêmes et sur leurs contextes plus que sur les individus qui les pratiquent. C'était en particulier le cas pour les données électorales, peu portées sur l'engagement en entreprise ${ }^{16}$, et pour les données de l'inspection du travail ou, plus récemment, pour celles de l'enquête $\mathrm{ACEMO}^{17}$ sur les conflits du travail. La différenciation des données de la statistique publique selon les modes d'action participait également d'un cloisonnement des analyses sur chaque forme de participation (le vote, la grève, la syndicalisation, etc.). Ce cloisonnement des enquêtes ou leur caractère parcellaire se retrouve au niveau international et dans de nombreux pays. En effet, les grandes enquêtes européennes ou internationales nous renseignent peu sur la participation au travail des salariés en dehors de leur adhésion à un syndicat, qui recouvre des significations très variables d'un pays à l'autre, et éventuellement de leur participation à une grève. Il en est de même dans la plupart des enquêtes nationales, même lorsqu'à l'image du Royaume-Uni des enquêtes spécifiques et riches ont été développées sur les relations professionnelles comme la Workplace Employement Relations Survey (WERS). Ainsi, le vote professionnel, qui prend des formes variables d'un système de relations professionnelles à l'autre, est analysé à l'aide de données agrégées ${ }^{18}$ ou de rares enquêtes d'opinion ${ }^{19}$. Ce découpage des objets, redoublé par la division du travail entre des spécialistes de sociologie électorale, des sociologues des relations professionnelles et des politistes des mobilisations collectives, ne permet pas d'établir un panorama de l'intensité et des inégalités de la participation en entreprise. En effet, on sait finalement peu de choses sur l'articulation des différentes formes et inégalités de participation

${ }^{14}$ Daniel Gaxie, Le cens caché. Inégalités culturelles et ségrégation politique, Paris, Seuil, 1978.

15 Carine Burricand, François Gleizes, «Trente ans de vie associative », INSEE Première, 1580, janvier 2016.

${ }^{16}$ Cette tradition est néanmoins en train d'évoluer, à l'instar de l'enquête électorale française de 2017 qui dispose de plusieurs questions sur ce sujet.

${ }^{17}$ Enquête « Activités et conditions d'emploi de la main-d'œuvre » (ACEMO) réalisée par la Dares.

${ }^{18}$ Dans le cas belge, voir Pierre Blaise, « Les élections sociales de mai 2016 », Courrier hebdomadaire du CRISP, 2333-2334, 2017, p. 5-62.

${ }^{19}$ Dans le cas espagnol, voir Miguel A. Malo, «Temporary Workers and Direct Voting Systems for Workers’ Representation », Economic and Industrial Democracy, 27 (3), 2006, p. 505-535. 
en entreprise : à l'image du champ politique, ceux qui votent le plus sont-ils également ceux qui sont le plus engagés dans d'autres formes de participation, notamment les plus revendicatives? Les pratiques de participation sont-elles fortement différenciées socialement selon leur nature et certains groupes sociaux sont-ils exclus de toutes formes de participation? Nous proposons dans cet article de dresser ce panorama en proposant une analyse conjointe des effets des déterminants sociaux et de la structuration du champ économique sur la participation en entreprise, restreinte ici aux formes de participation instituées par le droit du travail (élections professionnelles, droit de grève et de manifestation, libertés syndicales), et donc sans tenir compte de toutes les dimensions de la participation au travail définie comme l'implication des salariés dans les décisions relatives à leur travail et leur entreprise ${ }^{20}$.

Pour ce faire, nous nous appuierons sur les données du volet «salariés » de l'enquête «Relations professionnelles et négociation d'entreprise» (REPONSE, voir encadré 1). L'apport de cette source, jamais exploitée en ce sens, outre sa systématicité dans le temps et au sein du tissu productif français, est qu'elle renseigne sur la situation des salariés et leur (non)participation, tout du moins s'agissant des pratiques liées à la représentation du personnel et à l'action syndicale. Elle permet également de combiner l'analyse de l'influence des caractéristiques des salariés et celle des établissements dans lesquels ils exercent ${ }^{21}$, ce qui répond à la nécessité réaffirmée en sociologie électorale de désagréger les « variables lourdes » et, plus largement, de réencastrer les pratiques participatives dans leurs contextes de production. Nous pouvons alors jouer sur les deux niveaux, à l'échelle des salariés comme de l'établissement, pour saisir les articulations entre les différentes pratiques de participation déclarées par les salariés, en objectiver les déterminants sociaux et économiques et en restituer les évolutions récentes. C'est à la fois le caractère unique de ce type d'enquête mais aussi les spécificités des différentes pratiques de participation au travail étudiées qui nous conduisent d'ailleurs à limiter notre étude de cas à la France et plus particulièrement au secteur privé.

Il semble d'autant plus intéressant d'objectiver et d'analyser les inégalités de

${ }^{20}$ Nous ne prenons pas en compte, dans cet article, les dispositifs managériaux mis en place pour mobiliser au maximum la force de travail des salariés ou pour associer ceux-ci aux résultats financiers de l'entreprise. Sur une lecture de cette notion par des économistes, voir Patricia Crifo, Antoine Rebérioux, La participation des salariés. Du partage d'information à la codétermination, Paris, Presses de Sciences Po, 2019.

${ }^{21}$ Un questionnaire détaillé est passé indépendamment à un représentant de la direction et à un représentant du personnel de l'établissement des salariés. Toutefois, en moyenne seulement trois salariés ont répondu à l'enquête pour chaque établissement bien que ce chiffre varie, ce qui rend impossible toute modélisation multiniveau avec cette source comme avec les autres évoquées. 
participation en entreprise que plusieurs évolutions structurelles du champ économique ont vraisemblablement pu modifier les capacités de participer et de revendiquer en entreprise. Les séries statistiques manquent pour faire une analyse sur un temps long et l'enquête REPONSE ne permet de travailler au niveau individuel que depuis le début des années 2000. Cela n'interdit pas toutefois de poser quelques hypothèses. On sait en effet que, depuis les années 1990, la précarisation des conditions d'emploi et le développement de la sous-traitance concourent à rendre plus coûteuse la participation, au moins dans ses dimensions revendicatives ${ }^{22}$. La montée en puissance des travailleurs précaires pourrait en premier lieu obérer les capacités de participation des classes populaires qui sont plus concernées par les contrats temporaires, l'intérim et la sous-traitance. De même, le processus de déconcentration productive ${ }^{23} \mathrm{du}$ tissu économique français favorise l'augmentation des petites et moyennes entreprises qui sont moins favorables aux conditions requises pour la participation des salariés (moins d'institutions représentatives du personnel, moins de présence syndicale). Ce processus se combine avec une forte tertiarisation de l'économie française qui fragilise les conditions de mobilisation des classes populaires (déclin des bastions ouvriers dotés d'une tradition de mobilisation, précarisation des conditions d'emploi, développement de métiers considérés comme peu qualifiés et qui s'exercent de façon isolée comme ceux de femme de ménage, d'aide à domicile, de livreur, etc.). À l'inverse, cette dernière transformation concourt au développement du groupe des cadres et des professions intermédiaires, sûrement moins enclins à la mobilisation en entreprise, mais dont on peut aussi faire l'hypothèse que le poids plus important dans les entreprises encourage à se mobiliser et à participer pour faire valoir ses intérêts. De ce point de vue, il s'agit donc de repérer l'ampleur et les facteurs des inégalités de participation en entreprise en faisant l'hypothèse que les transformations que l'on vient de décrire ont en priorité affecté le potentiel de participation des classes populaires, mais de façon différenciée en leur sein.

Dans un premier temps, nous évaluerons l'intensité de la participation en entreprise, abordée en combinant plusieurs formes de participation (vote, syndicalisation, actions revendicatives, arrêt de travail, participation à des réunions organisées par les représentants du

${ }^{22}$ Paul Bouffartigue, Sophie Béroud (dir.), Quand le travail se précarise, quelles résistances collectives ?, Paris, La Dispute, 2009.

${ }^{23}$ Loup Wolff, « Des instances représentatives du personnel qui, malgré les évolutions du tissu productif, se maintiennent », dans Thomas Amossé, Catherine Bloch-London, L. Wolff (dir.), Les relations sociales en entreprise. Un portrait à partir des enquêtes "Relations professionnelles et négociations d'entreprise » [REPONSE 1992-1993, 1998-1999 et 2004-2005], Paris, La Découverte, 2008, p. 85-101. 
personnel). L'intérêt de l'enquête REPONSE est de ne pas réduire la participation au fait de déclarer participer ou ne pas participer, mais d'inclure une modalité de réponse indiquant ne pas avoir participé faute de cadre pour le faire (employeur n'ayant pas organisé d'élection, absence de réunion ou d'arrêt de travail organisé par un syndicat). On montre alors que l'exclusion de fait, autrement dit l'absence de cadres pour participer, est une composante décisive de la non-participation et qu'elle est complémentaire de ce qu'on peut appeler, à la suite d'autres travaux, l'auto-exclusion, inhérente au fait de ne pas participer alors que les conditions existent pour le faire.

Dans un deuxième temps, l'analyse portera sur les déterminants de l'exclusion de fait et de l'auto-exclusion. On montrera que, si cette dernière obéit à des déterminants sociologiques en lien avec les caractéristiques sociales et les conditions d'emploi et de travail des individus, la première est fortement corrélée aux logiques de fonctionnement du champ économique et aux différenciations entre les entreprises. Lorsque les conditions de participation sont présentes, il est possible d'identifier un «cens caché $^{24} »$, qui semble toutefois moins dépendant des inégalités en matière de qualification que dans le champ politique et avant tout lié au degré de précarité et d'intégration professionnelle des salariés. Ce parallèle doit aussi être nuancé par le fait que, pour toutes les pratiques, mais surtout en matière de conflictualité, on observe une auto-exclusion de la participation des cadres et des plus diplômés.

Enfin, dans un troisième temps, nous questionnerons les facteurs de la baisse tendancielle de toutes les formes de participation en entreprise depuis le début des années 2000 : s'expliquet-elle plutôt par l'exclusion de fait ou par l'auto-exclusion ? Nous verrons notamment que, si les mobilisations collectives déclinent légèrement, elles apparaissent surtout moins suivies par les salariés.

\section{Encadré 1. Le volet « salariés » de l'enquête REPONSE : apports et limites}

L'enquête REPONSE ${ }^{25}$, réalisée depuis 1993 par la Direction de l'animation de la recherche, des études et des statistiques (Dares) qui dépend du ministère du Travail, comprend un questionnaire s'adressant aux représentants de la direction et aux représentants du personnel ainsi que, depuis 1998, un questionnaire s'adressant aux salariés. Depuis 2011, l'enquête porte sur un échantillon représentatif des salariés des entreprises de 11 salariés et plus du secteur marchand et associatif non agricole, soit 9,7 des 19 millions de salariés que compte le secteur

\footnotetext{
${ }^{24}$ D. Gaxie, Le cens caché..., op. cit.

${ }^{25}$ Sur cette enquête, nous renvoyons à l'ouvrage de synthèse présentant les enjeux méthodologiques et certains résultats édité à la suite de l'édition 2005 : T. Amossé, C. Bloch-London, L. Wolff (dir.), Les relations sociales en entreprise..., op. cit.
} 
marchand et associatif en 2017. La procédure d'échantillonnage, du tirage aléatoire au calcul des pondérations, est stable d'une vague à l'autre, ce qui rend les données du volet « salariés » comparables, même si le taux de réponse a évolué : $24 \%$ en $2005(\mathrm{n}=11769), 33 \%$ en 2011 $(n=18536)$ et $61 \%$ en $2017(n=28963)^{26}$.

Depuis 2005, le questionnaire interroge les salariés sur leur adhésion syndicale et, durant les trois années précédant l'enquête, sur leur participation à un scrutin professionnel, à une réunion organisée par les représentants du personnel, à un arrêt collectif de travail ou à une autre forme d'action collective (manifestation, pétition, etc.). Il documente en parallèle les caractéristiques sociales des salariés ainsi que les contextes de l'établissement dans lesquels ils s'inscrivent, grâce à un appariement possible avec les données issues de l'interrogation indépendante d'un représentant de la direction (RD) et d'un du personnel (RP).

Cette enquête comporte certaines limites. D'abord, si elle nous renseigne sur le fait d'avoir pratiqué ou non tel ou tel mode d'action, elle n'informe ni sur sa fréquence, ni sur les orientations de l'action, et notamment du vote. De plus, l'enquête ne dit rien ou presque des pratiques des salariés en dehors de l'entreprise et de leur participation à des formes nouvelles ou suscitées par les employeurs comme le référendum ou le bénévolat en entreprise, ce qui nous contraint à définir de manière restrictive la participation en entreprise. Ensuite, si, comme toute enquête par questionnaire, elle est dépendante des déclarations des salariés, notons que le questionnaire n'étant envoyé qu'aux salariés présents dans l'établissement depuis plus d'un an, les salariés les plus précaires et en contrat à durée déterminée (CDD) courts sont exclus de l'enquête, tout comme les fonctionnaires. Enfin, l'édition 2005 de l'enquête ne portant que sur les salariés des établissements de 20 salariés et plus, nous sommes contraints de restreindre notre analyse à cette catégorie afin de comparer les données sur trois vagues et une période de près de 15 ans.

\section{Entre engagement revendicatif et exclusion de fait, formes et intensité de la} participation en entreprise

L'analyse des données de l'enquête REPONSE montre que les formes de participation ne sont pas utilisées dans des proportions identiques par tous les salariés et que la majorité d'entre eux participent peu. Cette faible participation s'explique en partie par l'absence de cadres d'action et de mobilisation en entreprise.

Participer en entreprise : un engagement majoritairement de faible intensité

${ }^{26}$ Le taux de réponse de l'enquête de 2017 est supérieur à celui de 2011 parce que l'enquête a acquis le statut d'enquête « obligatoire » par le Conseil national de l'information statistique (CNIS) et parce qu'une relance postale a été effectuée alors que cela n'avait pas été le cas en 2011. 
La participation des salariés en entreprise est inégalement distribuée dans le salariat selon les formes qu'elle prend. En effet, les différentes formes de participation impliquent des coûts matériels et humains inégaux.

Voter aux élections professionnelles est le mode de participation le plus partagé (voir tableau 1) : environ $67,1 \%$ des salariés déclarent avoir voté à des élections professionnelles entre 2014 et 2016. À l'opposé, seuls 6,5\% déclarent exercer un mandat de représentant du personnel ou de délégué syndical. Évidemment, les chances d'exercer un mandat de représentant sont restreintes dans la mesure où le nombre de «mandats » est limité par le volume de postes éligibles. Le nombre de salariés syndiqués est d'ailleurs supérieur à celui des «représentants » : 12,5\% des salariés déclarent être syndiqués en 2017. Le lien des salariés avec les syndicats s'opère, au-delà de l'adhésion, par le biais des réunions avec les élus du personnel : 23,5\% des salariés déclarent avoir participé au cours des trois années précédant l'enquête et 70,9\% des participants n'ont jamais été syndiqués.

\section{Tableau 1. Fréquences du recours à différentes formes de participation en entreprise}

\section{Formes de participation}

Être élu.e ou mandaté.e [variable indisponible]

Être syndiqué.e

Avoir participé à une grève
2008-2010

11,3

16,7

18,3
2014-2016

Avoir participé à une autre forme d'action collective

Avoir participé à une réunion organisée par les représentants du personnel

Avoir voté à un scrutin professionnel
81,1
67,1

Source : Dares, enquêtes REPONSE 2005, 2011 et 2017, volet «salariés » dans un établissement dont un représentant de la direction (RD) a été interrogé (appariement des volets « salariés » et $\mathrm{RD}$ ) ; données diffusées par l'Archive de données issues de la statistique publique (ADISP).

Champ : salariés des établissements de 20 salariés ou plus du secteur marchand et associatif non-agricole et travaillant depuis plus d'un an dans l'établissement; données pondérées par la Dares.

Lecture : sur la période 2014-2016, 12,4\% des salariés déclarent avoir participé à une grève.

Notes : le taux de syndicalisation, estimé ici à 12,5\% en 2017, est à prendre avec précaution car cette information est utilisée dans le calcul de pondération, mais il est du même ordre de grandeur que celui d'autres sources (environ 
$10 \%$ dans le privé, selon les enquêtes Statistiques sur les ressources et conditions de vie. Le taux de participation à un scrutin professionnel est également du même ordre de grandeur que celui constaté lors des élections avec présence syndicale dans les entreprises de 11 salariés ou plus entre 2013 et $2016(62,4 \%)$.

La pratique de la grève, du simple débrayage à la grève de plus de 48 heures, et la participation à des mobilisations sans arrêt de travail (pétition, rassemblement, manifestation, etc.) sont moins fréquentes : environ 12,4 \% des salariés déclarent avoir fait grève entre 2014 et 2016 et 13,6 \% affirment avoir participé à une autre forme d'action collective sans arrêt de travail. Minoritaire, l'expérience de la grève n'en reste pas moins un horizon d'action possible pour une part du salariat plus large que les seules «bases » syndicales, malgré le volume en apparence identique des deux populations. En effet, l'équation «syndicaliste égal gréviste » n'est vraie que pour $37,3 \%$ des syndiqués et pour seulement $36,6 \%$ des grévistes, tandis qu'à l'inverse $64,1 \%$ des grévistes ne sont pas syndiqués. Au final, cette première description de l'engagement dans l'entreprise indique qu'une majorité des salariés participe électoralement, mais qu'une minorité s'engage sur des pratiques plus coûteuses que sont la grève, la mobilisation revendicative et la syndicalisation.

Ce panorama est renforcé lorsque l'on prend en compte la combinaison des formes de participation. Pour préciser l'intensité de l'engagement en entreprise, nous avons observé les combinaisons existantes entre quatre indicateurs : l'adhésion syndicale, actuelle ou passée ; la participation à une grève ; la participation à une mobilisation collective sans arrêt de travail ; et la participation à l'élection de représentants du personnel ${ }^{27}$. Le nombre de configurations possibles est élevé (80) mais les cinq combinaisons les plus fréquentes concentrent $64 \%$ des salariés (voir tableau 2). Parmi celles-ci, trois se caractérisent par une absence totale de participation (représentant $25 \%$ des salariés) et deux (39\%) par le seul vote aux élections professionnelles. Le vote demeure donc le mode d'action le plus récurrent du fait de son faible coût, de son caractère anonyme et de l'organisation d'un scrutin dans la plupart des établissements. La grande majorité des salariés participent de façon résiduelle tandis que le cumul de modes d'action plus revendicatifs est plus rare puisque seule la septième combinaison la plus fréquente (3\% des salariés) correspond au recours à ce type de pratiques (participation à des conflits collectifs avec ou sans arrêt de travail, syndicalisation, et participation électorale). Si les 73 possibilités restantes ne correspondent qu'à $30 \%$ des répondants, elles constituent une «longue traîne » qui, si elle est difficile à interpréter, marque une diversité des profils de participation parmi les salariés «participants ».

${ }^{27}$ Nous excluons dorénavant la prise de mandat de représentant car elle est limitée par le nombre de postes éligibles ou désignés et ne peut donc pas constituer un horizon des possibles pour tous les salariés. 
Tableau 2. La « longue traîne » des engagements de salariés en entreprise

\begin{tabular}{|c|c|c|c|}
\hline $\mathbf{N}^{\mathbf{0}}$ & $\begin{array}{c}\text { Combinaisons les plus fréquentes de formes de } \\
\text { participation }\end{array}$ & $\begin{array}{c}\text { Fréquences dans } \\
\text { l'échantillon (\%) }\end{array}$ & $\begin{array}{c}\text { Pourcentages } \\
\text { cumulés (\%) }\end{array}$ \\
\hline 1 & $\begin{array}{c}\text { Absence de conflit collectif, salarié jamais syndiqué } \\
\text { mais votant }\end{array}$ & 29 & 29 \\
\hline 2 & $\begin{array}{c}\text { Absence de conflit collectif et d'élection, salarié } \\
\text { jamais syndiqué }\end{array}$ & 14 & 53 \\
\hline 3 & $\begin{array}{c}\text { Salarié ne participant pas aux conflits collectifs de } \\
\text { l'établissement, jamais syndiqué, mais votant }\end{array}$ & 7 & 60 \\
\hline 4 & $\begin{array}{c}\text { Absence de conflit collectif, salarié jamais syndiqué } \\
\text { et abstentionniste }\end{array}$ & 4 & 64 \\
\hline 5 & $\begin{array}{c}\text { Salarié ne participant pas aux conflits collectifs de } \\
\text { l'établissement, jamais syndiqué, abstentionniste }\end{array}$ & 3 & 67 \\
\hline 6 & Absence de conflit collectif, syndiqué et votant & 30 \\
\hline 7 & $\begin{array}{c}\text { Salarié engagé dans des conflits collectifs avec et } \\
\text { sans arrêt de travail, syndiqué et votant }\end{array}$ & 30 & 100 \\
\hline & Autres combinaisons (73 possibilités) & 3 & 67 \\
\hline
\end{tabular}

Source : Dares, enquête REPONSE 2017, volet « salariés » dans un établissement dont le RD a été interrogé (appariement des volets « salariés » et RD) ; données diffusées par l'ADISP.

Champ : salariés des établissements de 20 salariés ou plus du secteur marchand et associatif non agricole et travaillant depuis plus d'un an dans l'établissement ; données pondérées par la Dares.

«L'exclusion de fait », une composante déterminante de la non-participation

Une analyse des correspondances multiples (ACM) ${ }^{28}$ permet de structurer ces combinaisons et de faire ressortir les grands pôles de la participation en entreprise. La figure 1 présente les résultats des deux premiers axes, qui concentrent près de $40 \%$ de l'inertie, tandis que les contributions numériques des modalités sont en annexe (voir tableau 4).

${ }^{28}$ Nous avons inclus comme variables actives le recours des salariés aux quatre formes d'engagement considérées pour les éditions 2005 et 2011. Le nombre de répondants en 2017 étant beaucoup plus important (voir supra), une repondération périlleuse aurait été nécessaire pour les inclure en tant qu'individus actifs. Les répondants à l'édition 2017 de l'enquête sont donc projetés en individus supplémentaires. 
Figure 1. L'espace de la participation des salariés en entreprise

\section{Variables - Axes 1 \& 2}

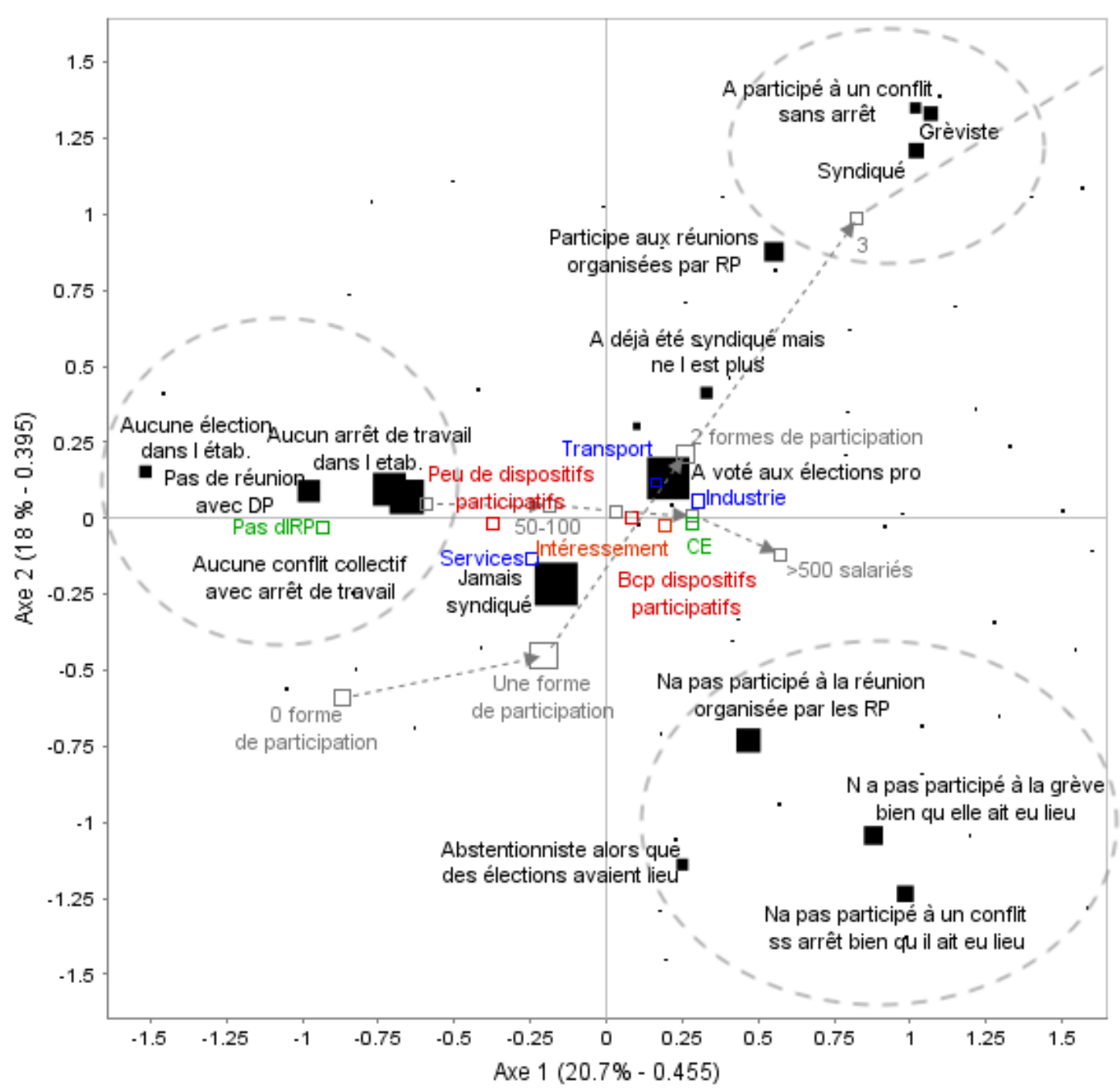

Source : Dares, enquêtes REPONSE 2005, 2011, 2017, volets « salariés » et « représentants de la direction »; données diffusées par l'ADISP.

Champ des individus actifs : répondants aux éditions 2005 et 2011 de l'enquête ; salariés des établissements de 20 salariés ou plus du secteur marchand et associatif non-agricole et travaillant depuis plus d'un an dans l'établissement.

Notes : les modalités des variables actives sont en noir; IRP : instance représentante du personnel ; CE : comité d'entreprise. Les dispositifs participatifs sont les groupes qualité ; les réunions d'atelier, de bureau, ou de service ; les groupes d'expression directe. La taille des points est proportionnelle aux effectifs concernés.

Les pratiques de participation au travail forment un continuum, à l'image de ce qui est observé pour la participation politique ${ }^{29} \mathrm{ou}$, au niveau agrégé de l'entreprise, entre la

${ }^{29}$ Olivier Fillieule, Stratégies de la rue. Les manifestations en France, Paris, Presses de Sciences Po, 1997, p. $127-162$. 
conflictualité et la négociation collective ${ }^{30}$. Dans ce continuum, le vote, qu'on retrouve au centre de la figure 1, est la pratique la moins distinctive.

Le premier axe résume l'information principale, à savoir l'opposition entre une minorité de salariés très actifs et revendicatifs et un groupe très important de salariés qui se trouvent exclus de fait de la participation. Ces derniers sont ceux qui, faute de conflit collectif organisé dans leur établissement ou, dans une moindre mesure, faute de scrutin organisé, ne sont tout simplement pas appelés à participer. Ce n'est que sur le second axe que se distinguent ceux qui participent beaucoup et ceux qui, bien qu'en ayant la possibilité, ne s'engagent pas.

Ainsi, l'alternative entre participer ou ne pas participer à un mode d'action ne résume pas entièrement les logiques de la participation en entreprise. En effet, pour participer, encore faut-il que l'action soit rendue possible sur le lieu de travail. Par exemple, il faut qu'un syndicat ait appelé à un arrêt de travail ou organisé une manifestation pour que les salariés puissent y participer. Dans ce cas, la non-participation à une grève est d'abord le fait de l'absence de grève sur le lieu de travail : en 2017, 60 \% des salariés déclarent qu'il n'y en a pas eu sur leur lieu de travail. Ils sont trois fois moins nombreux à refuser de faire grève alors qu'une grève a eu lieu (23\%). De même, 61,4\% des salariés déclarent ne pas avoir manifesté ou signé une pétition faute d'une occasion pour le faire quand seulement $21 \%$ d'entre eux n'ont pas souhaité le faire bien qu'ils le pouvaient effectivement. La participation est également dépendante de l'employeur s'agissant des scrutins professionnels. Certes, les établissements enquêtés sont normalement assujettis à l'obligation d'avoir au moins une institution représentative du personnel (IRP), mais tous les employeurs ne s'y conforment pas. Si des divergences entre salariés et directions peuvent exister, à l'image de ce qui a été observé entre représentants du personnel et des directions ${ }^{31}$, on note que $17,7 \%$ des salariés déclarent ne pas avoir eu l'occasion de participer à un scrutin ${ }^{32}$ et seulement $14,4 \%$ se sont effectivement abstenus alors qu'un scrutin a eu lieu.

L'absence de cadres permettant la participation produit une exclusion de fait qui peut se combiner avec de l'auto-exclusion : en 2017, la moitié des employeurs justifient l'absence de scrutin dans leur établissement par l'absence de candidature et un quart par l'absence de

\footnotetext{
${ }^{30}$ S. Béroud et al., La lutte continue ?, op. cit., p. 102.

${ }^{31}$ Pierre Blavier, Jérôme Pélisse, « Voir toujours midi à sa porte ? Divergences et désaccords entre acteurs des relations professionnelles en entreprises pendant les années 2000 », Sociologie, 10 (2), 2019.

${ }^{32}$ Pour rappel, le champ du volet «Salariés » de l'enquête REPONSE exclut les salariés présents dans l'établissement depuis moins d'un an ; ceux, donc, qui ont le plus de chances de ne pas avoir connu d'élections professionnelles.
} 
demande des salariés ${ }^{33}$. Il s'agit toutefois de comprendre si les déterminants de l'exclusion de fait et de l'auto-exclusion sont convergents ou s'ils concernent des profils de salariés ou d'entreprises différents.

\section{Les déterminants sociaux et économiques de la participation}

L'enquête met donc au jour une exclusion de fait, faute de possibilité de participer, et une auto-exclusion de la participation alors que celle-ci est possible. Ces deux processus ont des déterminants singuliers, même si certains salariés sont doublement exclus de la participation. D'un côté, les principaux facteurs de l'exclusion de fait des salariés de la participation en entreprise sont liés aux logiques de fonctionnement du champ économique. D'un autre côté, pour les salariés qui déclarent pouvoir participer, leur participation effective dépend principalement de leurs caractéristiques socioprofessionnelles. Toutefois, ces déterminants sociaux varient d'une pratique à l'autre.

L'exclusion de fait : un clivage interne au champ économique qui n'est pas neutre socialement

Le premier axe de l'ACM (voir figure 1), qui correspond à l'exclusion de fait, est fortement déterminé par les caractéristiques économiques de l'établissement des salariés. Les petits établissements, avec peu de salariés, sont très corrélés au pôle des exclus de fait, tandis qu'à l'inverse ceux avec plus d'effectifs sont rattachés à la présence de scrutin ou de grève. En matière électorale, 33,2 \% des salariés des établissements de 20 à 49 salariés déclarent qu'aucun scrutin n'a eu lieu alors qu'ils ne sont que 10,8 \% dans les établissements de 200 à 499 salariés et 7,2 \% dans les établissements de 500 salariés et plus. En matière de conflits collectifs, 78,3\% des salariés des établissements de 20 à 49 salariés ont déclaré une absence d'arrêt collectif de travail alors qu'ils ne sont que 49,8\% dans les établissements de 200 à 499 salariés et 37,7 \% dans les établissements de 500 salariés et plus. En complément de la taille de l'établissement, celle de l'entreprise apparaît également comme un facteur déterminant de la présence de conflit ou de scrutin. Ces résultats confirment les conclusions des travaux qui ont montré comment les relations professionnelles dans les petites entreprises (moins de 50 salariés) se traduisent par des relations interpersonnelles, par un dialogue social informel, par une personnalisation des

${ }^{33}$ Fabrice Romans, «Les relations professionnelles en 2017 : un panorama contrasté du dialogue social dans les établissements ?», Dares Analyses, 15, avril 2018. 
négociations salariales ou des conditions de travail et par un fonctionnement sur la base de micro-arrangements entre l'employeur et ses salariés plutôt que sur des formes de participation collectives et institutionnalisées. Si ce mode de gestion «paternaliste» ou «domestique » n'empêche pas certaines formes informelles de participation, il ne favorise ni l'émergence de conflits collectifs, ni l'organisation de scrutins ${ }^{34}$, autrement dit les formes les plus institutionnalisées de la participation en entreprise. À l'inverse, les relations professionnelles sont plus structurées dans les grandes entreprises, notamment celles appartenant à un groupe ou cotées en Bourse, recourant plus à de l'intéressement ou à des dispositifs participatifs (boîte à idées, groupe d'expression, charte, etc.) qui se projettent ainsi sur la droite du premier axe (voir figure 1).

Les effets de la taille des établissements se combinent avec leur appartenance sectorielle : les établissements du secteur tertiaire (commerces et services) et du bâtiment sont marqués par le manque d'occasions pour participer, à l'opposé des établissements du secteur des transports et de l'industrie (voir figure 1) qui connaissent traditionnellement plus de conflits mais aussi plus de scrutins. Ainsi, des enquêtes monographiques dans certains de ces secteurs ${ }^{35}$ ont démontré la difficulté de l'implantation syndicale du fait de l'atomisation du salariat et de la forte répression patronale, ce qui limite la conflictualité faute de cadres collectifs pour l'organiser.

Cette incidence des variables relatives aux logiques de fonctionnement du champ économique nous rappelle à quel point, comme le soulignait déjà John Dunlop en 1950, analyser les relations professionnelles nécessite de saisir le contexte économique, social et technique dans lequel elles sont produites ${ }^{36}$. À ce titre, dans le cas français, des travaux mobilisant le volet « représentants de la direction » de l'enquête REPONSE ont montré l'influence sur les relations professionnelles d'autres variables comme les politiques de gestion des ressources humaines ou

${ }^{34}$ Céline Dumoulin, «Quand les syndicats s'invitent dans les petites entreprises : les relations sociales dans les établissements de 11 à 49 salariés », La nouvelle revue du travail, 15, 2019, en ligne : http://journals.openedition.org/nrt/5817.

35 Nicolas Jounin, Chantier interdit au public. Enquête parmi les travailleurs du bâtiment, Paris, La Découverte, 2008 ; Marlène Benquet, Les damnées de la caisse. Enquête sur une grève dans un supermarché, Bellecombes-en-Bauges, Éditions du Croquant, 2011 ; Jean-Michel Denis, «"Dans le nettoyage, on ne fait pas du syndicalisme comme chez Renault!": implantation et stratégies syndicales dans le secteur du nettoyage industriel », Politix, 85, 2009, p. 105-126.

36 John T. Dunlop, William F. Whyte, «Framework of The Analysis of Industrial Relations : Two Views », ILR Review, 3 (3), 1950, p. 383-401. 
la structure de l'actionnariat ${ }^{37}$, soulignant la pertinence d'une analyse en termes de modèles socioproductifs $^{38}$. Or cette structuration du champ économique ${ }^{39}$ qui joue sur l'exclusion de fait n'est pas neutre socialement. En effet, la composition sociale de la main-d'œuvre n'est pas identique dans les petits et grands établissements. Par ce biais s'explique le fait que les ouvriers qualifiés de l'industrie se projettent du côté du pôle disposant de cadres de participation tandis que c'est l'inverse pour leurs homologues de l'artisanat (voir figure 2). De manière plus générale, en 2011, près de $17 \%$ des employés et des ouvriers déclarent l'absence de scrutin organisées les trois dernières années dans leur établissement contre $7 \%$ pour les cadres et $11 \%$ pour les professions intermédiaires (voir tableau 3). En matière de conflit collectif avec ou sans arrêt de travail dans l'établissement, $60 \%$ des employés et $53 \%$ des ouvriers déclarent leur absence dans leur établissement contre $44 \%$ pour les cadres et $42 \%$ pour les professions intermédiaires. Les classes populaires (ouvriers et employés) sont plus souvent exclues de fait de la participation parce que travaillant dans des espaces du champ économique où les conditions de cette participation (organisation de scrutins, de manifestations, d'arrêts de travail) ne sont pas réunies. Cette situation limite les potentialités de mobilisation de ces groupes sociaux alors que, lorsqu'ils peuvent participer, certains le font plus souvent que les cadres et professions intermédiaires.

${ }^{37}$ Thomas Philippon, Le capitalisme des héritiers. La crise française du travail, Paris, Seuil, 2007.

38 Thomas Amossé, Thomas Coutrot, « En guise de conclusion. L’évolution des modèles socioproductifs en France depuis 15 ans : le néo-taylorisme n'est pas mort», dans T. Amossé, C. Bloch-London, L. Wolff (dir.), Les relations sociales en entreprise, op. cit., p. 423-451.

${ }^{39}$ En particulier lorsqu'on tient compte des déclarations divergentes entre salariés et employeurs ; pour plus de précisions et pour montrer ces liens toutes choses égales par ailleurs, voir Tristan Haute, « Les salarié.e.s aux urnes », cité, p. 237-245. 
Tableau 3. Exclusion de fait et auto-exclusion selon la catégorie socioprofessionnelle

\begin{tabular}{|c|c|c|c|c|}
\hline $\begin{array}{c}\text { Conflit avec ou sans } \\
\text { arrêt de travail }\end{array}$ & A participé & Auto-exclusion & Exclusion de fait & Total \\
\hline Cadres & $17 \%$ & $39 \%$ & $44 \%$ & $100 \%$ \\
\hline PI & $30 \%$ & $28 \%$ & $42 \%$ & $100 \%$ \\
\hline Employées & $20 \%$ & $20 \%$ & $60 \%$ & $100 \%$ \\
\hline Ouvriers & $27 \%$ & $20 \%$ & $53 \%$ & $100 \%$ \\
\hline Ensemble & $24 \%$ & $25 \%$ & $51 \%$ & $100 \%$ \\
\hline $\begin{array}{c}\text { Élections } \\
\text { professionnelles }\end{array}$ & A participé & Auto-exclusion & Exclusion de fait & \\
\hline Cadres & $77 \%$ & $16 \%$ & $7 \%$ & $100 \%$ \\
\hline PI & $78 \%$ & $11 \%$ & $11 \%$ & $100 \%$ \\
\hline Employées & $71 \%$ & $11 \%$ & $18 \%$ & $100 \%$ \\
\hline Ouvriers & $72 \%$ & $11 \%$ & $17 \%$ & $100 \%$ \\
\hline Ensemble & $74 \%$ & $12 \%$ & $14 \%$ & $100 \%$ \\
\hline
\end{tabular}

Source : Dares, enquête REPONSE 2011, volets « salariés » et « représentants de la direction »; données diffusées par l'ADISP.

Champ : salariés des établissements de 20 salariés ou plus du secteur marchand et associatif non agricole et travaillant depuis plus d'un an dans l'établissement ; données pondérées par la Dares.

Lecture : en 2011, $60 \%$ des employés étaient exclus de fait de tout conflit collectif avec ou sans arrêt de travail, c'est-à-dire n'y ont pas pris part parce que leur établissement n'en a pas connu, tandis que $20 \%$ s'en sont autoexclus, c'est-à-dire n'y ont pas participé alors qu'une mobilisation a eu lieu dans leur établissement au cours des trois dernières années. En revanche, $20 \%$ se sont mobilisés. Le total de ces trois attitudes vis-à-vis de l'action collective fait donc $100 \%$.

L'auto-exclusion de la participation : des précaires aux cadres

Le second axe de l'ACM oppose les salariés ayant participé à des conflits collectifs et, dans une moindre mesure, à un scrutin professionnel à ceux qui n'ont pas eu recours à ces formes de participation bien qu'ils en aient eu la possibilité (voir figure 1). À chaque fois, les participations à un conflit avec ou sans arrêt de travail sont finalement très liées et concernent une minorité de salariés comme l'indique la taille des points de ces modalités sur le graphique. L'ancienneté, le niveau de rémunération et, dans une moindre mesure, la catégorie socioprofessionnelle vont dans le même sens et leur projection arquée (voir figure 2) permet de formuler le résultat suivant: ce sont avant tout les classes populaires stabilisées et les professions intermédiaires, en particulier les techniciens, qui se mobilisent tandis que les plus précaires et les moins qualifiés tout comme les cadres participent moins. Pour ces derniers, leur positionnement au plus près des directions d'entreprise dans le travail d'encadrement de la main-d'œuvre peut fournir une clé d'explication. Toutefois, on observe que les cadres participent tout aussi peu dans les établissements où ils sont majoritaires, autrement dit dans les 
entreprises où leur fonction d'encadrement est secondaire, voire marginale.

Ce constat général gagne à être précisé en isolant, d'une part, les effets propres toutes choses égales par ailleurs de certaines variables et en opérant, d'autre part, des distinctions plus fines entre groupes socioprofessionnels. 
Figure 2. Espace de la participation et caractéristiques sociales des salariés

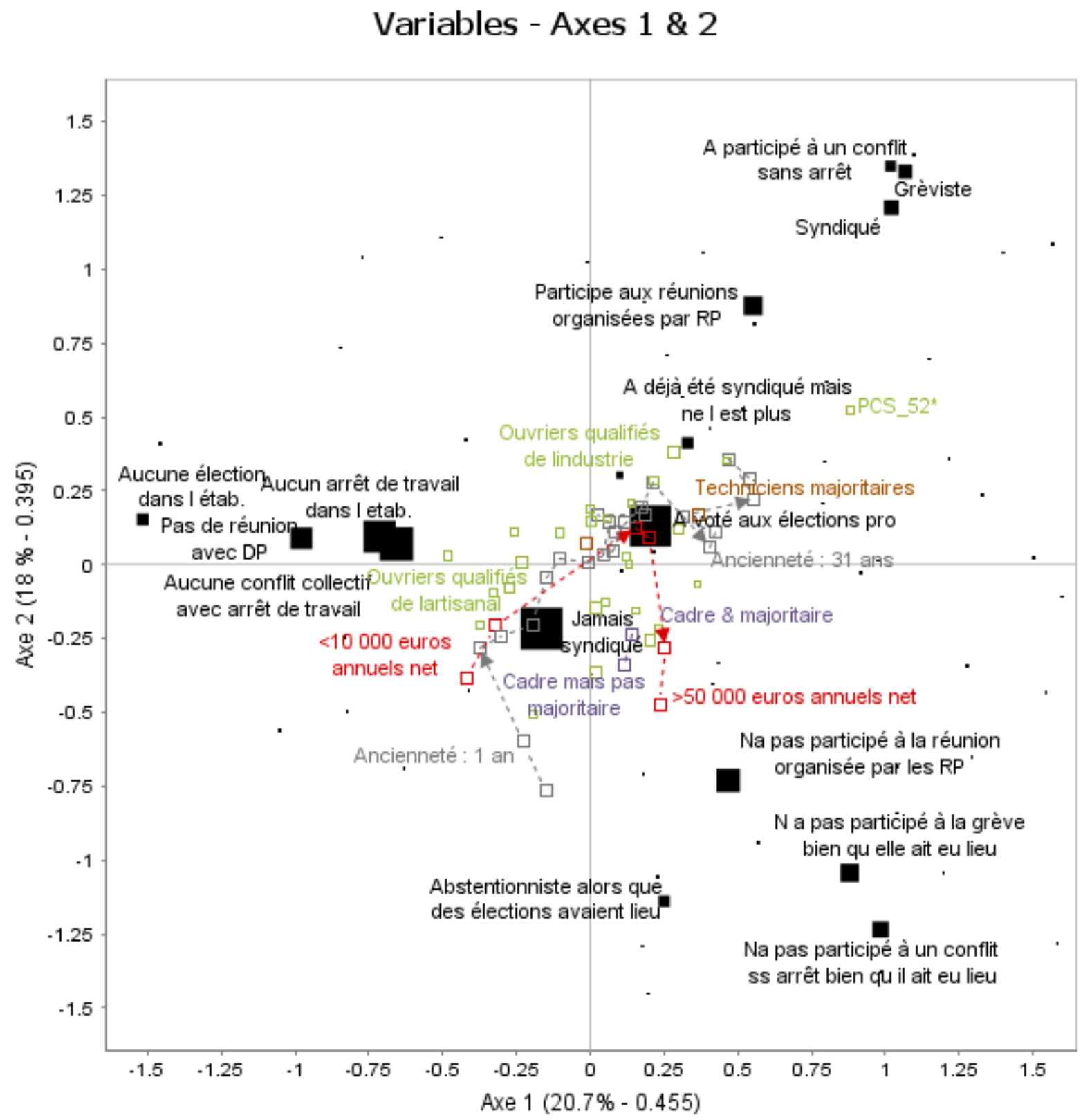

Source : Dares, enquêtes REPONSE 2005, 2011, 2017, volets «salariés » dans un établissement dont un représentant de la direction (RD) a été interrogé (appariemment des volets « salariés » et RD).

Champ : salariés des établissements de 20 salariés ou plus du secteur marchand et associatif non agricole et travaillant depuis plus d'un an dans l'établissement, données pondérées par la Dares.

Note : pour plus de lisibilité, les modalités supplémentaires projetées au centre du graphique (année d'enquête, indicateurs de conflictualité individuelle tels que recours aux prud'hommes ou aux sanctions, sexe, certaines PCS, etc.) ont été retirées. PCS 52* : « employés civils et agents de service de la fonction publique » qui, dans l'enquête REPONSE 2011, sont majoritairement des agents de service hospitalier du privé (31\%) aides-soignantes (27\%), et aides médico-psychologiques $(15 \%)$ du secteur privé. 


\section{Encadré 2. Modélisation de l'auto-exclusion du vote et de la grève}

Nous avons, à partir des données 2017 de l'enquête REPONSE, modélisé les chances, pour un salarié ayant déclaré qu'un scrutin professionnel avait eu lieu ces trois dernières années dans son établissement, de s'être abstenu et, pour un salarié ayant déclaré qu'un arrêt collectif de travail avait eu lieu ces trois dernières années dans son établissement, de ne pas y avoir participé. Les variables indépendantes sont le sexe, l'âge, le niveau de diplôme, l'ancienneté dans l'établissement, la position professionnelle déclarée par le salarié, le fait d'être à temps partiel ou non, le fait d'avoir obtenu ou non une promotion ces trois dernières années, le fait de travailler ou non dans un collectif de travail stable (toujours ou souvent avec les mêmes collègues), le type d'horaires (fixes ou variables), le nombre d'heures de travail par semaine, le niveau de satisfaction dans le travail, le fait d'être autonome ou non dans son travail (défini comme le fait de ne pas avoir d'objectifs chiffrés et de résoudre toujours ou souvent soi-même les incidents éventuels), le fait d'être syndiqué ou non, le fait d'exercer un mandat de représentant du personnel ou non, la taille de l'établissement, la taille de l'entreprise, le statut de l'établissement, la structure de l'actionnariat et le secteur d'activité. Les pondérations de la Dares ont été normalisées ${ }^{40}$. Les résultats des modèles sont présentés en annexe (voir tableau 5).

Des cadres en retrait des modes de participation collective

Toutes choses égales par ailleurs, plusieurs variables sociodémographiques « classiques » en matière de participation politique jouent un rôle déterminant. Ainsi les salariés les plus jeunes s'abstiennent davantage, confirmant l'hypothèse d'un «moratoire ${ }^{41}$ ». De même, les salariées (16,3\%), lorsqu'elles ont la possibilité de voter, ont une propension légèrement moins importante que les hommes $(18,3 \%)$ à ne pas mettre effectivement un bulletin dans l'urne ${ }^{42}$. À l'inverse, elles font significativement moins grève (29\% contre 38,6\% des hommes). Ce dernier résultat est à mettre en lien avec le coût en temps et en argent de la participation gréviste dans un contexte où l'articulation entre activités syndicales, professionnelles et familiales demeure difficile pour les salariées ${ }^{43}$.

Combiné à ces variables sociodémographiques, le niveau d'intégration dans l'entreprise

${ }^{40} \mathrm{Si}$ on réalise les régressions logistiques présentées dans cet article sans tenir compte des pondérations, les résultats obtenus sont très proches en termes de significativité et de rapports de chance.

${ }^{41}$ Pour reprendre l'expression d'Anne Muxel, également utilisée par plusieurs chercheurs s'agissant du rapport des jeunes au syndicalisme. Voir Sophie Béroud et al., «Jeunes et engagements au travail : une génération asyndicale ? », Agora Débats/Jeunesses, 82, 2019, p. 7-25.

${ }^{42}$ Céline Braconnier, Baptiste Coulmont, Jean-Yves Dormagen, « Toujours pas de chrysanthèmes pour les variables lourdes de la participation électorale : chute de la participation et augmentation des inégalités électorales au printemps 2017 », Revue française de science politique, 67 (6), décembre 2017, p. 1023-1040.

${ }^{43}$ Cécile Guillaume, Syndiquées. Défendre les intérêts des femmes au travail, Paris, Presses de Sciences Po, 2018. 
et dans les collectifs de travail influence fortement la participation électorale des salariés. Selon les données de l'enquête REPONSE 2017, lorsqu'ils sont confrontés à un scrutin, les salariés à temps partiel $(22,3 \%)$ ou n'ayant pas de collectif de travail stable $(24,2 \%)$ ont plus de chances de s’y abstenir que les salariés à temps complet (16,8\%) ou travaillant toujours ou souvent avec les mêmes collègues (16,6\%). De même, les salariés ayant une faible ancienneté ont plus de chances de s'abstenir que les salariés les plus anciens. Ces résultats s'inscrivent dans la continuité de nombreux travaux relatifs aux scrutins dans la fonction publique ${ }^{44}$, aux scrutins prud'homaux $^{45}$ ou aux élections syndicales en Espagne ${ }^{46}$. Ils sont conformes à ce qui est observé en matière de participation aux scrutins «politiques ${ }^{47}$ ». S'agissant de la participation gréviste, c'est moins le degré d'intégration professionnelle que le rapport subjectif au travail qui constitue une variable structurante : lorsqu'ils sont confrontés à une grève, les salariés très insatisfaits $(59,2 \%)$ et plutôt insatisfaits (44\%) dans leur travail y participent significativement plus que les salariés plutôt satisfaits $(33,3 \%)$ ou très satisfaits $(22,1 \%)$. En outre le poids des variables économiques mentionnées plus haut demeure non négligeable en matière de conflictualité : la participation gréviste est bien plus faible dans les petites entreprises, dans les établissements indépendants et dans les entreprises familiales.

Enfin, dans le cas du vote comme de la grève, les cadres participent significativement moins que les ouvriers qualifiés. Ce résultat converge avec le fait que les salariés les mieux rémunérés ${ }^{48}$, les plus diplômés (niveau bac +5 ou plus) et travaillant plus de 40 heures par semaine sont aussi moins engagés dans l'action revendicative. Cette moindre mobilisation des couches supérieures du salariat renvoie en premier lieu à leur statut dans l'entreprise, puisque ces salariés sont intégrés aux équipes de direction. Les cadres sont souvent pris dans une « relation salariale de confiance ${ }^{49} »$, en particulier quand ils sont minoritaires dans leur

44 Tristan Haute, «Le vote professionnel, entre rapport au syndicalisme et rapport au métier et à l'institution : le cas des enseignant.e.s du secteur public en France », Participations, 25, 2019, p. 139-164.

${ }^{45}$ Françoise Subileau, «Les élections prud'homales : participation, représentativité, légitimité », Politix, 47, 1999, p. 151-166.

${ }^{46}$ M. A. Malo, «Temporary Workers and Direct Voting System... », art. cité.

${ }^{47}$ Camille Peugny, Pour une prise en compte des clivages au sein des classes populaires. La participation politique des ouvriers et des employés», Revue française de science politique, 65 (5-6), octobre-décembre 2015 , p. 735-759, ici p. 745 .

${ }^{48}$ Cette variable n'étant pas disponible à l'ADISP pour l'édition 2017, les calculs ont été effectués à partir des données 2011.

${ }^{49}$ Paul Bouffartigue, Les cadres. Fin d'une figure sociale, Paris, La Dispute, 2001. 
établissement. Ils peuvent défendre leurs intérêts autrement que par l'intermédiaire d'une représentation collective du personnel à laquelle ils sont peu socialisés, privilégiant des canaux individuels ou des sociabilités professionnelles à part. Cette plus faible mobilisation des cadres contraste fortement avec leur fort engagement associatif ${ }^{50}$.

Si les variations de participation en fonction des propriétés sociales, notamment en matière électorale, ne sont pas sans lien avec un certain «cens caché », au sens où les compétences et le sentiment d'auto-habilitation à participer dépendent des caractéristiques des individus, on mesure qu'elles s'articulent surtout avec la manière dont les individus s'insèrent dans des groupes d'appartenance plus ou moins cohésifs (intégration professionnelle, stabilité du collectif de travail et plus ou moins grande autonomie face à l'employeur, présence syndicale) et modelés par les structures juridiques et économiques (institutionnalisation ou non des relations professionnelles, mode de gestion de la main-d'œuvre), ceci d'autant plus que le niveau de diplôme en soi n'apparaît pas, en matière de participation au travail, comme une variable structurante. Ceci se repère d'autant mieux qu'on descend à l'échelle plus fine des groupes socioprofessionnels.

La grève : d'importantes distinctions selon les groupes socioprofessionnels intermédiaires et populaires

En effet, prendre en compte un niveau plus détaillé des groupes socioprofessionnels ${ }^{51}$ conduit à identifier des contrastes non négligeables (voir figure 3) sur les chances de participation. Nous considérons ici la participation effective, c'est-à-dire quand elle est possible, des salariés aux scrutins professionnels et aux grèves selon leur catégorie socioprofessionnelle (PCS) à deux chiffres. Les cadres, du moins ceux du champ de l'enquête, apparaissent nettement moins mobilisés lors des grèves, qui demeurent plutôt l'arme des professions intermédiaires, des employés et des ouvriers, mais d'une partie seulement. En effet, la pratique de la grève pour construire un rapport de force avec l'employeur reste la marque distinctive des fractions industrielles du monde ouvrier (techniciens, ouvriers qualifiés de type industriel et manutentionnaires) et des transports. À l'inverse, les ouvriers qualifiés et non qualifiés de type artisanal apparaissent moins enclins à voter et à faire grève, ce qui montre que l'opposition principale dans la participation est moins liée à la qualification, contrairement à

\footnotetext{
${ }^{50}$ Carine Burricand, François Gleizes, « Trente ans de vie associative... », art. cité.

${ }^{51}$ Cédric Hugrée, « Les sciences sociales face à la mobilité sociale : les enjeux d'une démesure statistique des déplacements sociaux entre générations », Politix, 114, 2016, p. 47-72.
} 
d'autres formes de participation associative ou culturelle par exemple ${ }^{52}$, qu'aux univers de travail et aux secteurs d'appartenance. La grève est également une pratique de participation importante parmi les salariés des services parapublics, en particulier des professions intermédiaires de la santé et du travail social et, dans une moindre mesure, des employés de grandes entreprises publiques (la Poste, ex-France Télécom, SNCF, etc.) bien que leurs effectifs soient de plus en plus restreints. Le clivage est manifeste entre les professions intermédiaires et les employés qui exercent des métiers « sociaux » dans des secteurs parapublics ou fortement liés à l'État et ceux qui exercent des métiers administratifs et commerciaux. Ces résultats convergent avec ceux de Camille Peugny en matière de participation électorale ${ }^{53}$, du moins pour les franges comparables entre les données utilisées par l'auteur, restreintes aux employés et aux ouvriers de la population générale, et ceux de REPONSE, qui excluent le secteur agricole et la fonction publique ${ }^{54}$. Nous retrouvons un net décalage entre les ouvriers de type industriel, très mobilisés, et ceux de type artisanal qui le sont beaucoup moins, en entreprise comme dans le champ politique. En revanche, les personnels des services directs aux particuliers sont moins engagés dans les grèves (voir figure 3) alors que C. Peugny montre qu'ils sont étonnamment peu abstentionnistes. Cela peut s'expliquer par un certain isolement professionnel et des collectifs de travail qui peinent à se structurer syndicalement ${ }^{55}$. À l'inverse, les ouvriers nonqualifiés de l'industrie sont plus grévistes que le reste des salariés alors qu'ils apparaissent dans la moyenne pour leur participation électorale. Ces différences se vérifient surtout pour les grèves, beaucoup moins pour la participation aux élections professionnelles qui demeure moins clivante socialement.

${ }^{52}$ Thomas Amossé, Olivier Chardon, «Les travailleurs non qualifiés : une nouvelle classe sociale ? », Économie et Statistique, 393-394, 2006, p. 203-229.

${ }^{53}$ C. Peugny, « Pour une prise en compte des clivages... », art cité, tableau 5, p. 749.

${ }^{54}$ Ceci empêche la comparaison entre certaines de ses analyses et les nôtres, comme pour la catégorie de PCS 53 «policiers et militaires » qui, dans notre cas, ne correspond en réalité qu'aux agents de sécurité.

${ }^{55}$ Sur ces difficultés, voir, par exemple, Christelle Avril, «Une mobilisation collective dans l'aide à domicile à la lumière des pratiques et des relations de travail », Politix, 86, 2009, p. 97-118; Sophie Béroud, « Une campagne de syndicalisation au féminin : une expérience militante dans le secteur de l'aide à domicile », Travail, genre et sociétés, 30, 2013, p. 111-128. 
Figure 3. Taux de participation effective aux élections professionnelles et aux grèves selon la PCS à deux chiffres (2011)

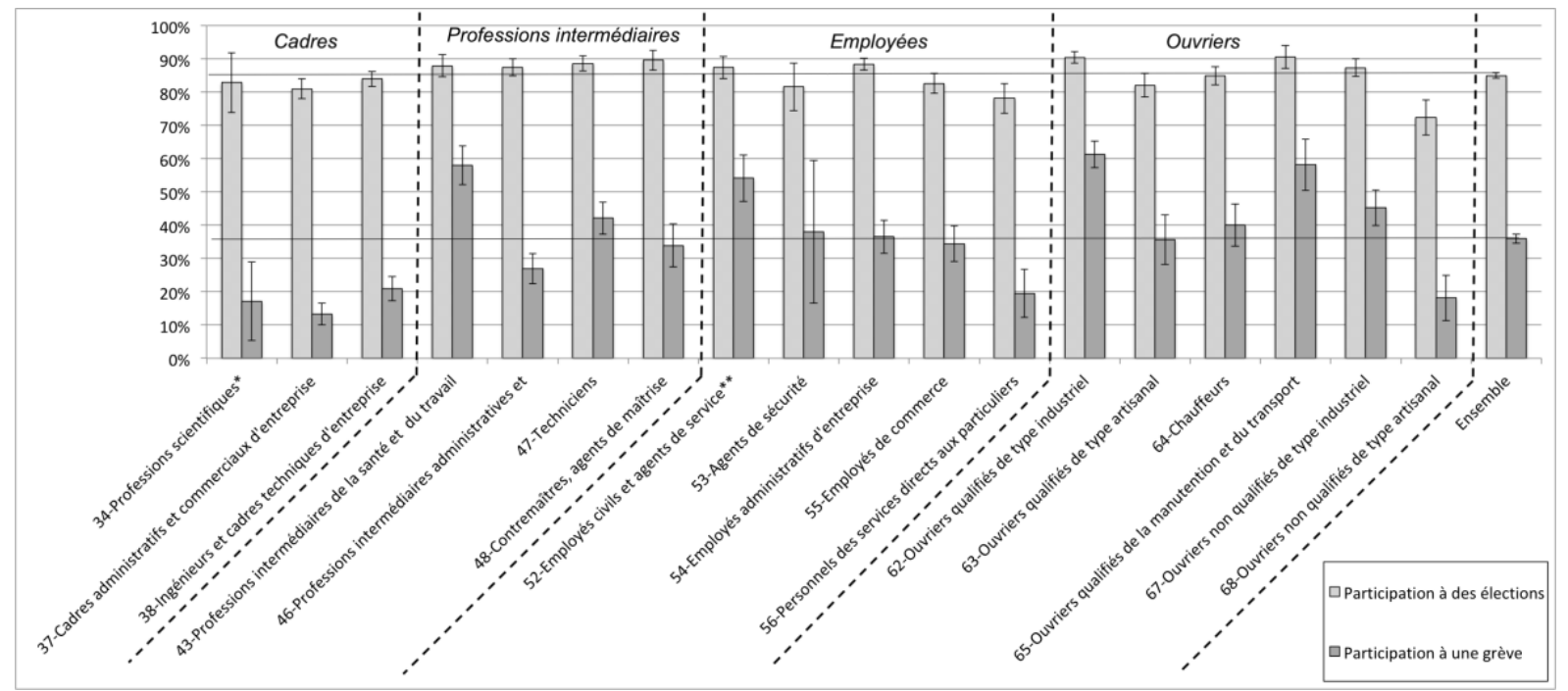

Source : Dares, enquête REPONSE 2011, volet « salarié » $\mathrm{N}=18536$.

Champ : salariés des établissements de 20 salariés ou plus du secteur marchand et associatif non agricole, travaillant depuis plus d'un an dans l'établissement et dont l'établissement a connu respectivement une élection professionnelle $(n=14918)$ ou une grève $(n=7438)$ au cours des trois dernières années $(2008,2009,2010)$. Du fait que les fonctionnaires, les intermittents et les indépendants, dont les professions libérales et les agriculteurs, soient hors-champ de l'enquête REPONSE, certaines PCS n'apparaissent pas ici. * La PCS 34 «professions scientifiques » correspond normalement aux professeurs et professions scientifiques mais, dans le champ REPONSE qui exclut la fonction publique, il s'agit surtout de pharmaciens et médecins salariés. ** La PCS 52 « employés civils et agents de service » correspond principalement aux agents hospitaliers, ambulanciers et aidessoignants du privé et, dans une moindre mesure, aux employés de la poste.

Lecture : dans les établissements ayant connu une grève, $20 \%$ des personnels des services directs aux particuliers disent y avoir participé contre $60 \%$ des ouvriers industriels qualifiés. Les intervalles de confiance au seuil de $5 \%$ visent à prendre en compte les variations d'effectifs de ces catégories et donc de qualités d'estimation des taux de participation. Ainsi, on a $95 \%$ de chances de ne pas se tromper en estimant que le pourcentage se situe entre les deux extrémités des intervalles. Si ceux-ci ne se chevauchent pas, l'écart entre les deux pourcentages peut être jugé statistiquement significatif.

\section{Déclin tendanciel de la participation : la faute à l'auto-exclusion ?}

L'exploitation des trois dernières éditions de l'enquête REPONSE permet de mettre au jour plusieurs évolutions sur une quinzaine d'années (2002-2016). En effet, la participation en entreprise semble avoir décliné sur cette période, en particulier entre 2011 et 2016 : cela s'explique-t-il par l'exclusion de fait ou par l'auto-exclusion? Autrement dit, ce déclin est-il lié à l'absence même de mobilisation à l'échelle des établissements ou bien au choix de ne pas y participer ? Parallèlement, une analyse plus fine des évolutions fait apparaître des soubresauts de la participation à des conflits collectifs dans certaines franges du salariat.

Une augmentation caractérisée de l'auto-exclusion 
Si les évolutions présentées dans le tableau 1 sont à considérer avec prudence car elles reposent sur la comparabilité des trois vagues d'enquête, elles vont dans le sens d'une nette diminution de la participation en entreprise qui marque tous les modes de participation entre le début des années 2000 et le milieu des années 2010. La participation électorale (- 14 points) mais également l'engagement dans la grève ( -4 points) et les autres formes d'actions revendicatives ( -5 points) baissent sensiblement, tout comme la participation à une réunion organisée par des représentants du personnel (-4,6 points $)^{56}$.

Cette tendance est d'autant plus sensible qu'elle ne tient pas uniquement à l'absence de scrutin ou de conflit à l'échelle des établissements. En effet, la figure 4 nous montre que, d'après les salariés ${ }^{57}$, l'occurrence de conflits collectifs a certes reculé ces dernières années, mais légèrement et après un rebond de la conflictualité dans l'enquête de 2011. Ce sont surtout les taux de mobilisation des salariés dans ces conflits qui ont continûment diminué : les taux de mobilisation agrégés pour les scrutins et pour les conflits avec et sans arrêt de travail sont passés de respectivement $90 \%, 41 \%$ et $50 \%$ en 2005 à $82 \%, 34 \%$ et $38 \%$ en 2017 . L'auto-exclusion semble donc augmenter de manière assez nette entre les deux dates : si les mobilisations et dispositifs collectifs déclinent légèrement, ils apparaissent surtout moins suivis par les salariés, même si les déterminants de la participation s'avèrent plutôt stables au cours des trois enquêtes. Sous cet angle, on mesure la difficulté des syndicalistes moins à proposer des cadres de mobilisation collective qu'à entraîner avec elles et eux les salariés dans ces mobilisations. Ce déclin des taux de participation s'inscrit dans un contexte de récession économique à partir de 2008 qui, s'il entraîne des mouvements sociaux en réponse aux plans de licenciements et de restructuration $^{58}$, ne favorise pas la participation massive des salariés. Et ce d'autant plus que cette période correspond à une dégradation des conditions d'emploi et de travail par le double effet de la crise économique et des politiques publiques de flexibilisation du marché du travail, dont nous avons montré qu'elle obère les chances de participer, en particulier au sein des classes populaires. Enfin, même s'il faudrait des questions sur le rapport subjectif aux modes de participation pour asseoir cette hypothèse, nul doute que l'intériorisation des défaites sociales,

\footnotetext{
${ }^{56}$ Pour la pratique de la grève, ces résultats convergent avec ceux issus de l'enquête SRCV (Insee). Voir
} Pierre Blavier, Tristan Haute, Étienne Pénissat, «La grève : entre soubresauts et déclin », Mouvements, 2020, à paraître.

${ }^{57}$ Cette tendance est corroborée à l'échelle des établissements.

${ }^{58}$ Baptiste Giraud, Jérôme Pélisse, Étienne Pénissat, « Gérer, négocier, résister : une approche dynamique des politiques patronales et des conflits au travail (des années 2000 à aujourd'hui) », rapport de recherche, Darès, novembre 2014. 
que ce soit lors des mobilisations interprofessionnelles de 2010 contre la réforme des retraites ou lors de mouvements localisés contre les fermetures d'usine ${ }^{59}$ ou les plans de licenciements ${ }^{60}$, contribue à la démonétisation de la participation en entreprise, notamment dans ses formes les plus protestataires.

\section{Figure 4. Évolution d'occurrence d'une mobilisation et taux de participation}

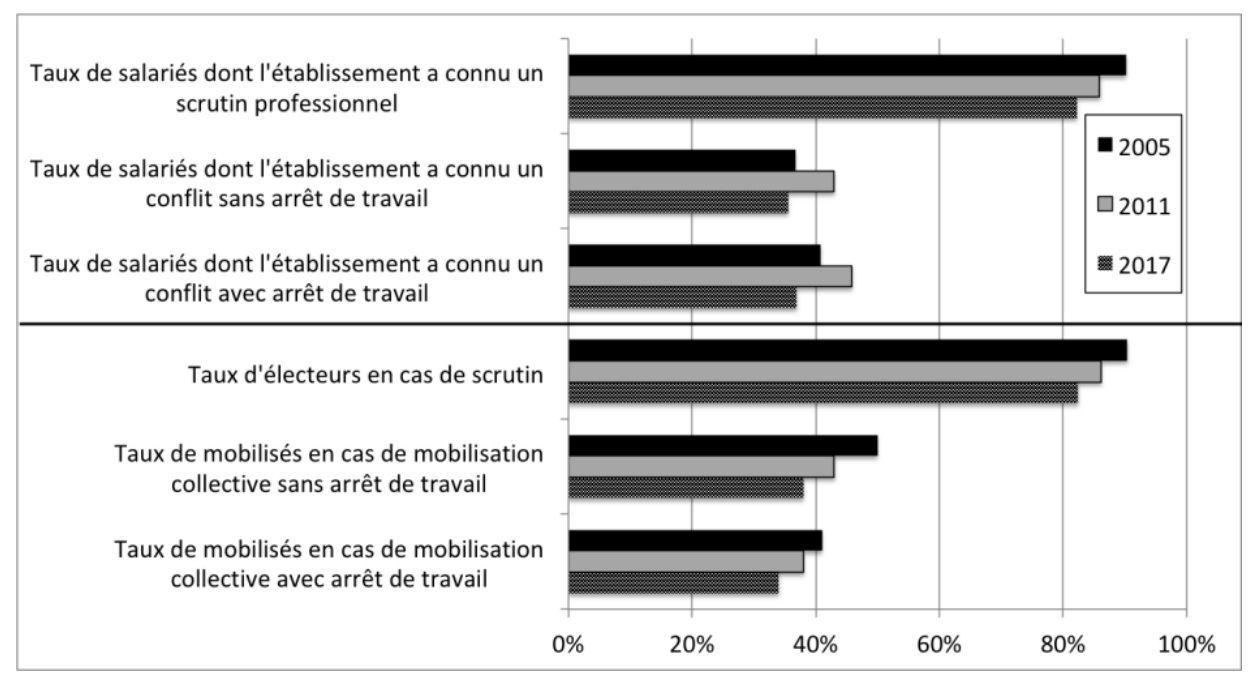

Source : Dares, enquêtes REPONSE 2005, 2011, 2017, volets « salariés » et « représentants de la direction »; données diffusées par l'ADISP.

Champ : salariés des établissements de 20 salariés ou plus du secteur marchand et associatif non agricole et travaillant depuis plus d'un an dans l'établissement; données pondérées par la Dares.

Lecture : en 2005 (barre en noir), $40 \%$ des salariés déclarent que leur établissement a connu au moins un conflit avec arrêt de travail au cours des trois dernières années (qu'ils y aient ou non participé). Parmi eux, $41 \%$ déclarent y avoir participé. En 2017, ce taux n'est plus que de $34 \%$ : les arrêts de travail, lorsqu'il y en a eu au moins un, sont en moyenne moins suivis par les salariés.

Au-delà du déclin, des soubresauts de participation pour certains groupes socioprofessionnels

Ce déclin de la participation en entreprise doit toutefois être nuancé, du moins en ce qui concerne les conflits. Nous avons en effet reproduit les taux de participation pour les éditions de 2005 à 2017 de l'enquête REPONSE, en les différenciant selon les catégories socioprofessionnelles des salariés. Pour la plupart des PCS, la participation décline, notamment

${ }^{59}$ Collectif du 9 août, Quand ils ont fermé l'usine. Lutter contre la délocalisation dans une économie globalisée, Marseille, Agone, 2017.

${ }^{60}$ Baptiste Giraud, Jérôme Pélisse, Étienne Pénissat, « Adapting While Resisting : The Ambivalence of Union Action in The Face of Lean Production », Sociologia Del Lavoro, 151, 2018, p. 193-209. 
entre 2011 et 2017. On observe à la fois un déclin des possibilités de participation et de la participation effective, que synthétisent les taux de participation à un conflit présentés dans la figure 5. Ce déclin est prononcé et linéaire pour les ouvriers qualifiés (PCS 62) comme nonqualifiés (PCS 67) de type industriel, bien qu'ils demeurent la catégorie sociale la plus gréviste, ce qui confirme une crise de démobilisation dans ce secteur en déclin et frappé par de nombreux plans de licenciements.

Toutefois, certains groupes professionnels sont marqués par une hausse des possibilités de participation, voire de la participation effective. Ainsi les employées du secteur sanitaire et social (PCS 52) ${ }^{61}$ ainsi que les ouvriers qualifiés de la manutention, du magasinage et du transport (PCS 65, qui intègre les ouvriers de la logistique $)^{62}$ ont à la fois plus de possibilités de participer à des actions collectives et y participent effectivement davantage. Leurs taux de syndicalisation se maintiennent à des niveaux élevés, dans les deux cas supérieurs à $15 \%$. Ces résultats correspondent à des évolutions professionnelles et à une conflictualité accrue dans ces secteurs. Ce constat est d'autant plus intéressant s'il est mis en lien avec la composition sociologique des Gilets jaunes, dans lesquels l'enquête collective nationale par questionnaire a pu montrer que ces deux secteurs sont particulièrement bien représentés ${ }^{64}$. Inversement, certains groupes comme les personnels des services directs aux particuliers (PCS 56) et dans une moindre mesure les agents de sécurité (PCS 53) participent davantage aux actions collectives, alors même que celles-ci sont moins nombreuses. Si on retient cette participation effective comme un indice de cohésion relative des groupes sociaux, eu égard à ces dispositifs de défense de leurs intérêts de salariés, on peut considérer que ces deux groupes professionnels commencent à s'affirmer comme tels, alors que cela semblait jusqu'ici « improbable ». Cela est corroboré par des taux de syndicalisation qui tendent à augmenter (entre 2008 et 2016, de $22 \%$

${ }^{61}$ Carlotta Benvegnù, « Dans les ateliers de la circulation. Une ethnographie du travail logistique entre le Grand Paris et la métropole diffuse vénitienne », thèse de doctorat en sociologie, Cédric Lomba, Devi Sacchetto (dir.), université Paris VIII, 2018.

${ }^{62}$ Emmanuelle Puissant, Laurent Gardin, Nadine Richez-Battesti, « Propos introductifs : syndicalisme et dialogue social dans l'aide à domicile », La Revue de l'IRES, 78, 2013, p. 3-24 ; Sophie Divay, Soignantes dans un hôpital local. Des gens de métier confrontés à la rationalisation et à la précarisation, Rennes, Presses de l'EHESP, 2013 ; Sophie Béroud, « Une campagne de syndicalisation au féminin... », art. cité.

${ }^{63}$ Source : enquête SRCV, Insee, 2008-2016. Celle-ci comprend les salariés et les fonctionnaires, donc ces chiffres ne sont pas directement comparables avec ceux de l'enquête REPONSE.

${ }^{64}$ Collectif d'enquête sur les Gilets Jaunes, « Enquêter in situ par questionnaire sur une mobilisation : une étude sur les Gilets jaunes », Revue française de science politique, 69 (5-6), octobre-novembre 2019, p. 869-892. 
à $25 \%$ pour la PCS 53, de $2 \%$ à $6 \%$ pour la PCS 56). 
Figure 5. Taux de participation à un conflit avec ou sans arrêt selon la PCS à deux chiffres, 2005-2017

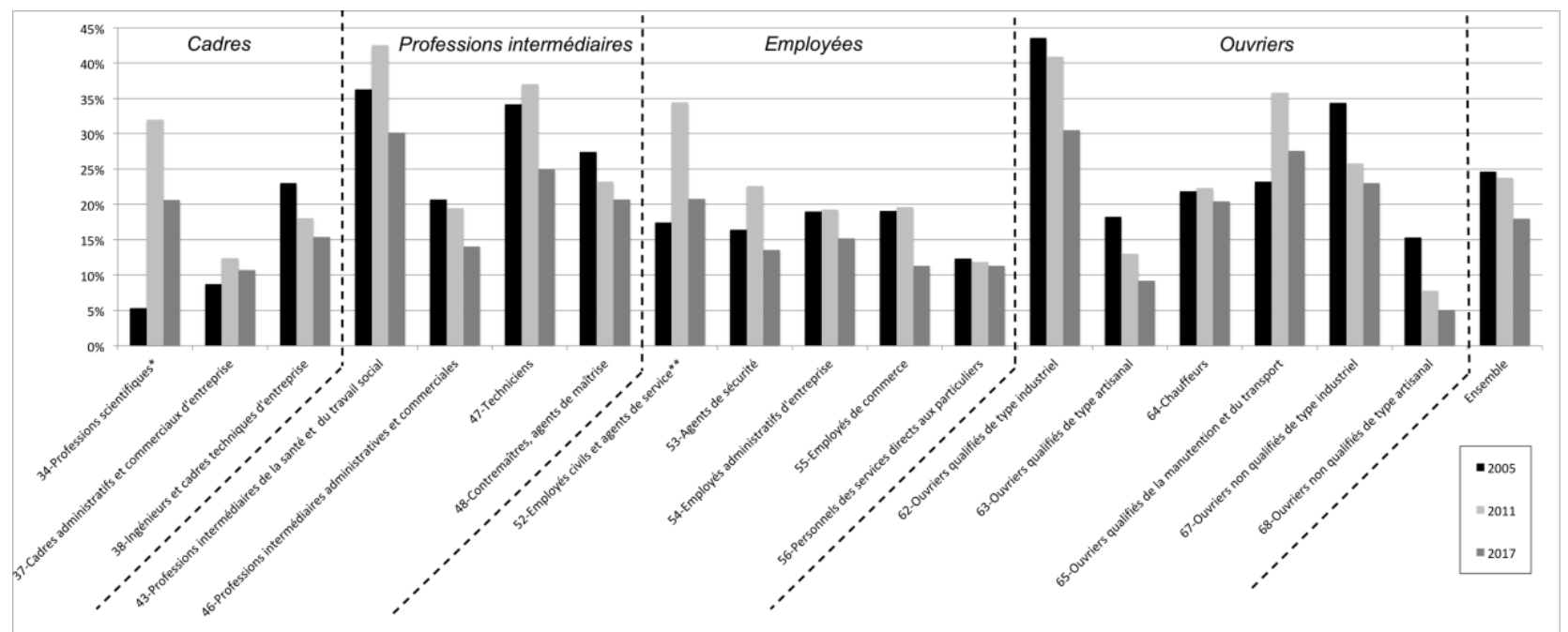

Source : Dares, enquêtes REPONSE 2005, 2011, 2017, volet « salariés »; données diffusées par l'intermédiaire du Centre d'accès sécurisé aux données (CASD, ANR-10-EQPX-17).

Champ : salariés des établissements de 20 salariés ou plus du secteur marchand et associatif non agricole, travaillant depuis plus d'un an dans l'établissement.

Lecture : en 2005 (barre en noir), près de $35 \%$ des salariés appartenant à la PCS 47 « Techniciens » déclarent avoir participé à au moins un conflit avec ou sans arrêt de travail au cours des trois dernières années.

\section{Conclusion}

Cette contribution sur la participation en entreprise permet de poser plusieurs résultats. Tout d'abord, les écarts de participation entre salariés résultent d'une articulation entre exclusion de fait et auto-exclusion, et ce quelle que soit la forme de mobilisation dont il s'agit (scrutin, syndicalisation, conflits, réunions, etc.). Si les possibilités de participation sont fortement dépendantes des contextes socioproductifs, c'est aussi à ce niveau que se joue indirectement une partie non négligeable des inégalités sociales de participation.

En se focalisant sur ceux qui peuvent participer, on montre que les plus précaires et les moins intégrés professionnellement s'auto-excluent de la participation, en particulier lors des élections professionnelles, confirmant que la précarisation des contrats de travail et les réformes visant à renforcer la mobilité et le turn-over des salariés risquent de nuire encore au niveau de participation des salariés et d'accroître les inégalités sociales en la matière. Mais, à rebours d'une lecture en termes de «cens caché », les salariés les plus hauts dans la hiérarchie, cadres et diplômés du supérieur, ne sont pas les plus mobilisés, même en matière électorale. Ce retrait participatif des classes supérieures dans l'entreprise peut être lié au faible rôle accordé à ces modes de participation dans la fabrication des décisions de l'entreprise, les élus du personnel 
étant consultés mais n'ayant pas ou peu de pouvoir de décision, et au maintien d'une frontière sociale entre les cadres et le reste du salariat quand ils se côtoient au sein d'un même établissement. En revanche, ce sont surtout les professions intermédiaires des services sociaux et de santé et les fractions industrielles du monde ouvrier qui sont les plus actives et les plus mobilisées pour faire élire des représentants et surtout pour construire un rapport de force par la grève avec leurs employeurs. Ce contre-exemple au regard des travaux sur la participation politique des classes populaires indique que la non-participation politique est à mettre en lien avec les contextes d'engagements, la perception des ressources dont disposent les acteurs sociaux pour faire valoir leurs intérêts et la plus ou moins forte cohésion des groupes sociaux auxquels ils appartiennent. Ainsi, les moins participatifs dans le champ politique ne le sont pas nécessairement dans des espaces économiques.

Enfin, la participation des salariés évolue globalement à la baisse depuis le début des années 2000, du fait d'une auto-exclusion accrue. Ce résultat est marqué pour la participation gréviste mais également pour le vote professionnel alors même que plusieurs lois relatives au dialogue social lui ont accordé un rôle plus important pour déterminer la représentativité des syndicats et leur pouvoir de négocier des accords d'entreprise et de branche. Ces dispositifs légaux ne s'accompagnent pas d'une dynamique participative. L'affaiblissement du pouvoir syndical et la multiplication des mobilisations se soldant par des défaites ont sûrement contribué aussi à démonétiser ces formes d'action. Ces éléments nourrissent donc l'hypothèse d'une projection des mobilisations concernant des revendications propres au monde du travail en dehors des établissements, notamment dans le cas des Gilets jaunes, du fait de la dévitalisation de la représentation syndicale et de la quasi-impossibilité pour certaines fractions du salariat, que l'on retrouve dans ce mouvement, de se faire entendre dans l'entreprise. Ainsi, ces mobilisations connaîtraient une politisation d'autant plus forte, sous la forme d'une interpellation directe des pouvoirs publics, que les possibilités de changement dans l'entreprise s'en trouvent réduites. Pour autant, les mobilisations sur le lieu de travail et en dehors ne s'opposent pas. Elles seraient complémentaires en permettant à des fractions différentes des classes populaires de faire entendre leur revendication ${ }^{65}$. D'ailleurs, la participation de certains groupes socioprofessionnels ${ }^{66}$ à ces deux formes d'action indique que des circulations s'opèrent entre les deux. Ces observations plaident pour approfondir le double travail de décloisonnement des enquêtes entre participation dans et hors de l'entreprise et d'investigation des

\footnotetext{
${ }^{65} \mathrm{~K}$. Yon, « Le syndicalisme, la retraite et les grèves... », art. cité.

${ }^{66}$ Collectif d'enquête sur les Gilets jaunes, « Enquêter in situ par questionnaire... », art. cité.
} 
différenciations des formes de participation et de mobilisation selon les appartenances sociales.

Pierre Blavier, chargé de recherche CNRS, travaille sur les inégalités de conditions de vie en Europe ainsi que sur les relations professionnelles et sur certains mouvements sociaux contemporains. Il a récemment publié, avec Jérôme Pélisse, « Voir toujours midi à sa porte ? Divergences et désaccords entre acteurs des relations professionnelles en entreprises pendant les années 2000 », Sociologie, 10 (2), 2019 ; «Les réaménagements de la consommation en contexte de récession », Revue française de sociologie, 59 (1), 2018, p. 7-36; avec Collectif d'enquête, «Déclassement sectoriel et rassemblement public: éléments de sociographie de Nuit Debout place de la République », Revue française de science politique, 67 (4), août 2017, p. 675-693; avec Frédéric Lebaron, «Classes et nations: quelle articulation à l'échelle européenne ? », Actes de la recherche en sciences sociales, 219, 2017, p. 80-97 (CLERSÉ, Bâtiment SH2, Cité scientifique, 59655 Villeneuve-d'Ascq, <pierre.blavier@univ-lille.fr>).

Tristan Haute, docteur en science politique, a récemment soutenu une thèse portant sur les ressorts des votes des salariés aux élections professionnelles dans le secteur privé en France. Il participe également à divers projets collectifs de recherche en sociologie de l'engagement, en sociologie des relations professionnelles et en sociologie électorale. Il a récemment publié : «Le vote professionnel, entre rapport au syndicalisme et rapport au métier et à l'institution : le cas des enseignant.e.s du secteur public en France », Participations, 25, 2019, p. 139-164 ; avec Karel Yon, «Comprendre la construction sociale de la représentativité syndicale : un éclairage à partir des branches professionnelles », Travail et emploi, 154, 2018, p. 101-124 ; «Évolutions du paysage syndical et du salariat : analyse des élections aux comités d'entreprise », La Revue de l'IRES, 94-95, 2018, p. 57-89 (CERAPS, 1 place Déliot, CS 10629, 59024 Lille Cedex, < tristan.haute@univ-lille.fr>).

Étienne Pénissat, chargé de recherche CNRS, travaille sur les classes sociales en Europe ainsi que sur l'engagement syndical et les formes d'entrave et de domestication de l'action collective en entreprise. Il a récemment publié : avec Cédric Hugrée, Alexis Spire, Social Class in Europe. New Inequalities in The Old World, Verso, 2020 ; avec Baptiste Giraud, Jérôme Pélisse, «Adapting While Resisting: The Ambivalence of Union Action in The Face to Lean Production », Sociologia del Lavoro, 151, 2018, p. 193-209 ; avec Cédric Hugrée, Alexis Spire, Classes sociales en Europe. Tableau des nouvelles inégalités sur le vieux continent, Agone, 2017 ; avec Baptiste Giraud, Amaël Marchand, «Le sentiment de discrimination des représentants du personnel : une étude à partir des données statistiques et monographiques liées à l'enquête REPONSE », Travail et Emploi, 145, 2016, p. 87-119 (CERAPS, 1 place Déliot, 
CS 10629, 59024 Lille cedex, <etienne.penissat@univ-lille.fr>). 


\section{Annexes}

Tableau 4. Contribution et coordonnées des modalités sur les deux premiers axes

\begin{tabular}{|c|c|c|}
\hline Axe 1 & - & + \\
\hline $\begin{array}{c}\text { Aucune grève dans l'établissement au cours des trois } \\
\text { dernières années }\end{array}$ & $17 \%$ & \\
\hline Aucun conflit collectif sans arrêt de travail & $16 \%$ & \\
\hline Aucune élection dans l'établissement & $13 \%$ & $13 \%$ \\
\hline A participé à un conflit sans arrêt & & $12 \%$ \\
\hline Gréviste & & $9 \%$ \\
\hline Non-gréviste bien qu'une grève ait eu lieu & & $9 \%$ \\
\hline $\begin{array}{c}\text { N'a pas participé à un conflit sans arrêt bien qu'il ait } \\
\text { eu lieu }\end{array}$ & & \\
\hline
\end{tabular}

\begin{tabular}{|c|c|c|}
\hline Axe 2 & - & + \\
\hline $\begin{array}{c}\text { N'a pas participé à un conflit sans arrêt bien qu'il ait } \\
\text { eu lieu }\end{array}$ & $22 \%$ & \\
\hline Non-gréviste bien qu'une grève ait eu lieu & $21 \%$ & \\
\hline Gréviste & & $18 \%$ \\
\hline A participé à un conflit sans arrêt & & $16 \%$ \\
\hline Abstentionniste alors qu'un scrutin a eu lieu & $9 \%$ & \\
\hline
\end{tabular}

Source : ACM présentée dans la figure 1.

Lecture : la modalité «absentionniste alors qu'un scrutin a eu lieu » compte pour $9 \%$ de l'inertie de l'axe 2, et a une coordonnée négative (voir figure 1.

Tableau 5. Non-participation à un scrutin et à une grève selon les caractéristiques des salariés et des établissements confrontés à ces pratiques (enquête REPONSE 2017)

\begin{tabular}{|l|l|l|l|l|l|}
\hline Variable & Modalité & $\begin{array}{l}\text { Abstention à } \\
\text { un scrutin }\end{array}$ & $\begin{array}{l}\text { Probabilité de } \\
\text { s'abstenir } \\
\text { (rapport de } \\
\text { chance) }\end{array}$ & $\begin{array}{l}\text { Non- } \\
\text { participation à } \\
\text { une grève pas faire }\end{array}$ & $\begin{array}{l}\text { Probabilité de } \\
\text { grève (rapport } \\
\text { de chance) }\end{array}$ \\
\hline Sexe & homme & 18,3 & ref. & 61,4 & ref. \\
\hline & femme & 16,3 & $0,74 * * *$ & 71 & $1,23 *$ \\
\hline Âge & moins de 25 ans 39 & $1,88^{* * *}$ & 80,8 & $n .5$. \\
\hline & 25 à 34 ans & 25,9 & $1,64 * * *$ & 698,6 & $0,75^{*}$ \\
\hline & 35 à 44 ans & 15,4 & 1,15 & 63,4 & $0,81^{*}$ \\
\hline & 45 à 54 ans & 12 & ref. & 64 & ref. \\
\hline
\end{tabular}




\begin{tabular}{|c|c|c|c|c|c|}
\hline & 55 ans ou plus & 11,4 & n.s. & 60 & n.s. \\
\hline \multirow[t]{7}{*}{ Diplôme } & Aucun & 20,1 & $1,45^{* *}$ & 60,3 & n.s. \\
\hline & $\begin{array}{l}\text { brevet ou } \\
\text { certificat d'étude }\end{array}$ & 13,1 & n.s. & 57,7 & n.s. \\
\hline & CAP ou BEP & 13,7 & n.s. & 56 & n.s. \\
\hline & Baccalauréat & 17,1 & n.s. & 59,8 & n.s. \\
\hline & $\mathrm{Bac}+2$ & 15,5 & ref. & 64,5 & ref. \\
\hline & $\begin{array}{l}\mathrm{Bac}+3 \text { ou } \\
\mathrm{bac}+4\end{array}$ & 18 & n.s. & 73,5 & n.s. \\
\hline & $\mathrm{Bac}+5$ ou plus & 24,8 & $1,29 *$ & 83 & $1,42 *$ \\
\hline \multirow[t]{6}{*}{ Emploi } & $\begin{array}{l}\text { Manœuvre ou } \\
\text { ouvrier non } \\
\text { qualifié }\end{array}$ & 18,7 & $1,34 * *$ & 50,8 & n.s. \\
\hline & Ouvrier qualifié & 13,5 & ref. & 50,6 & ref. \\
\hline & Employé & 17,5 & n.s. & 68,4 & $1,72^{* * *}$ \\
\hline & Technicien & 14 & n.s. & 65 & $1,83 * * *$ \\
\hline & $\begin{array}{l}\text { Cadre ou } \\
\text { ingénieur }\end{array}$ & 19,3 & n.s. & 81,6 & $2,33 * * *$ \\
\hline & Autre & 22,9 & $1,44 * *$ & 63,4 & n.s. \\
\hline \multirow[t]{2}{*}{ Temps partiel } & Non & 16,8 & ref. & 64,3 & ref. \\
\hline & Oui & 22,3 & $1,32$. & 70,5 & n.s. \\
\hline \multirow[t]{4}{*}{ Ancienneté } & 3 ans ou moins & 41,6 & $3,24 * * *$ & 83 & $1,71^{* *}$ \\
\hline & 4 à 9 ans & 22,3 & $1,51 * * *$ & 70,1 & n.s. \\
\hline & 10 à 14 ans & 13,9 & ref. & 63,2 & ref. \\
\hline & 15 ans ou plus & 9,4 & $0,77 * *$ & 58,2 & $0,70 * *$ \\
\hline \multirow{2}{*}{$\begin{array}{l}\text { Collectif de } \\
\text { travail stable }\end{array}$} & Oui & 16,6 & ref. & 64,9 & ref. \\
\hline & Non & 24,2 & $1,37 * * *$ & 66 & n.s. \\
\hline \multirow{2}{*}{$\begin{array}{l}\text { A obtenu une } \\
\text { promotion ces } 3 \\
\text { dernières années }\end{array}$} & Non & 17,4 & ref. & 63,5 & ref. \\
\hline & Oui & 17,8 & n.s. & 68,4 & n.s. \\
\hline \multirow[t]{2}{*}{ Horaires } & Fixes & 16,3 & ref. & 64,5 & ref. \\
\hline & Variables & 19,7 & n.s. & 66,3 & $0,80 * *$ \\
\hline \multirow{3}{*}{$\begin{array}{l}\text { Nombre } \\
\text { d'heures de } \\
\text { travail par } \\
\text { semaine }\end{array}$} & Moins de 35 & 21,4 & n.s. & 66,5 & n.s. \\
\hline & 35 à 40 heures & 15,9 & ref. & 59,8 & ref. \\
\hline & $\begin{array}{l}\text { Plus de } \\
40 \text { heures }\end{array}$ & 20 & $1,17^{*}$ & 84 & $2,53 * * *$ \\
\hline
\end{tabular}




\begin{tabular}{|c|c|c|c|c|c|}
\hline Autonomie dans & Non & 18 & ref. & 64,1 & ref. \\
\hline & oui & 16,3 & n.s. & 67,4 & n.s. \\
\hline Satisfaction dans & très satisfait & 19,7 & n.s. & 77,9 & $1,71^{* * *}$ \\
\hline & plutôt satisfait & 16,9 & ref. & 66,7 & ref. \\
\hline & plutôt insatisfait & 17,7 & n.s. & 56 & $0,69 * * *$ \\
\hline & très insatisfait & 19,1 & n.s. & 40,8 & $0,40 * * *$ \\
\hline Est syndiqué.e & non & 19,3 & ref. & 72,2 & ref. \\
\hline & oui & 6,2 & $0,44 * * *$ & 36,7 & $0,32 * * *$ \\
\hline & non & 18,8 & ref. & 67,6 & ref. \\
\hline & oui & 1,6 & $0,13^{* * *}$ & 31 & $0,45^{* * *}$ \\
\hline Taille de & 20 à 49 salariés & 16,2 & n.s. & 66,6 & 0,68 \\
\hline & 50 à 99 salariés & 14,3 & $0,68^{* * *}$ & 65,1 & n.s. \\
\hline & $\begin{array}{l}100 \text { à } \\
199 \text { salariés }\end{array}$ & 17,1 & n.s. & 64,3 & n.s. \\
\hline & $\begin{array}{l}200 \text { à } \\
499 \text { salariés }\end{array}$ & 17,8 & ref. & 63,5 & ref. \\
\hline & $\begin{array}{l}500 \text { salariés ou } \\
\text { plus }\end{array}$ & 21,5 & $1,39 * * *$ & 65,9 & $1,26 *$ \\
\hline Taille de & 20 à 49 salariés & 16,2 & n.s. & 82,1 & $2,68 * *$ \\
\hline & $\begin{array}{l}50 \text { à } \\
299 \text { salariés }\end{array}$ & 15,4 & 0,87 & 67,1 & $1,24 *$ \\
\hline & $\begin{array}{l}300 \text { salariés ou } \\
\text { plus }\end{array}$ & 18,7 & ref. & 63,6 & ref. \\
\hline Secteur d'activité & éBâtiment & 19,3 & n.s. & 75,9 & $1,61^{*}$ \\
\hline & Commerce & 16,1 & $0,73^{* * *}$ & 77,3 & $1,81^{* * *}$ \\
\hline & Industrie & 13,1 & $0,68^{* * *}$ & 57,5 & $0,71 * *$ \\
\hline & Services & 20,5 & ref. & 70,8 & ref. \\
\hline & Transports & 17,8 & n.s. & 54,5 & n.s. \\
\hline $\begin{array}{l}\text { Actionnaire } \\
\text { majoritaire }\end{array}$ & $\begin{array}{l}\text { Particulier ou } \\
\text { famille }\end{array}$ & 18 & ref. & 67,8 & ref. \\
\hline & $\begin{array}{l}\text { Société non } \\
\text { financière }\end{array}$ & 16 & n.s. & 65,5 & $0,64 * * *$ \\
\hline & $\begin{array}{l}\text { Organisme } \\
\text { financier }\end{array}$ & 15,9 & n.s. & 61 & 0,78 \\
\hline & $\begin{array}{l}\text { État ou } \\
\text { collectivité locale }\end{array}$ & $e^{14,6}$ & $0,64 * *$ & 54,3 & $0,41 * * *$ \\
\hline & Salariés & 17,8 & n.s. & 56,3 & 0,59 \\
\hline & $\begin{array}{l}\text { Inconnu ou } \\
\text { autre }\end{array}$ & 18,6 & n.s. & 68,5 & n.s. \\
\hline
\end{tabular}




\begin{tabular}{|l|l|l|l|l|l|}
\hline $\begin{array}{l}\text { Statut de } \\
\text { l'établissement }\end{array}$ & Indépendant & 18,9 & ref. & 72 & ref. \\
\hline & Franchisé & 19,1 & n.s. & 69,3 & n.s. \\
\hline & $\begin{array}{l}\text { Appartenant à } \\
\text { un groupe }\end{array}$ & 16,8 & n.s. & 62,8 & $0,82 *$ \\
\hline $\begin{array}{l}\text { Inconnu ou } \\
\text { autre }\end{array}$ & 16,2 & 0,56 & 69,4 & n.s. \\
\hline Ensemble & & 17,5 & & 65 & 0,21 \\
\hline PseudoR2 & & & 0,11 & & 7067 \\
\hline $\begin{array}{l}\text { Effectif de } \\
\text { l'échantillon }\end{array}$ & & & 15798 & & \\
\hline
\end{tabular}

Source : Dares, enquête REPONSE 2017, appariemment volets « salariés » et « représentants de la direction »; données diffusées par l'ADISP.

Champ : salariés des établissements de 20 salariés ou plus du secteur marchand et associatif non agricole, travaillant depuis plus d'un an dans l'établissement et ayant déclaré avoir été confrontés à un scrutin (colonnes 3 et 4) ou à une grève (colonnes 5 et 6 ); données pondérées par la Dares ; les non-réponses sont exclues.

Notes : $* * *=$ significatif au seuil de $0,1 \% ; * *=$ significatif au seuil de $1 \% ; *=$ significatif au seuil de $5 \%$; .$=$ significatif au seuil de $10 \% ;$ n.s. $=$ non significatif $;$ ref. $=$ modalité de référence.

Lecture : $16,3 \%$ des femmes confrontées à un scrutin ces trois dernières années se sont abstenues de voter contre $18,3 \%$ des hommes. Les femmes ont, toutes choses égales par ailleurs et de manière statistiquement significative (au seuil de 0,1\%), par rapport aux hommes, 0,74 fois plus de chances de s'être abstenues plutôt que d'avoir participé. 